\title{
HELLS and PRDM9 form a pioneer complex to open chromatin at meiotic recombination hot spots
}

\author{
Catrina Spruce, Sibongakonke Dlamini, Guruprasad Ananda, Naomi Bronkema, Hui Tian, \\ Kenneth Paigen, Gregory W. Carter, and Christopher L. Baker \\ The Jackson Laboratory, Bar Harbor, Maine 04660, USA
}

Chromatin barriers prevent spurious interactions between regulatory elements and DNA-binding proteins. One such barrier, whose mechanism for overcoming is poorly understood, is access to recombination hot spots during meiosis. Here we show that the chromatin remodeler HELLS and DNA-binding protein PRDM9 function together to open chromatin at hot spots and provide access for the DNA double-strand break (DSB) machinery. Recombination hot spots are decorated by a unique combination of histone modifications not found at other regulatory elements. HELLS is recruited to hot spots by PRDM9 and is necessary for both histone modifications and DNA accessibility at hot spots. In male mice lacking HELLS, DSBs are retargeted to other sites of open chromatin, leading to germ cell death and sterility. Together, these data provide a model for hot spot activation in which HELLS and PRDM9 form a pioneer complex to create a unique epigenomic environment of open chromatin, permitting correct placement and repair of DSBs.

[Keywords: H3K4me3; chromatin remodeling; germ cells; histone modification; meiosis; nucleosome-depleted region; pioneer factor]

Supplemental material is available for this article.

Received October 4, 2019; revised version accepted December 27, 2019.

In eukaryotic cells, most DNA is wrapped around an octamer of histone proteins to form nucleosomes, the basic unit of chromatin. Chromatin functions as a physical barrier to DNA, and regulating access to DNA is an essential aspect to normal cellular function. Numerous proteins have evolved to carry out this function; these include pioneer factors, chromatin remodelers, and epigenetic modifiers (Iwafuchi-Doi and Zaret 2014; Clapier et al. 2017). Pioneer factors initiate formation of accessible chromatin by engaging partial recognition motifs present within nucleosomal DNA (Soufi et al. 2012; Zaret and Mango 2016). Binding of the pioneer factor leads to increased accessibility accompanied by deposition of active histone modifications, ultimately enabling other DNA-binding proteins to gain access to regulatory elements (IwafuchiDoi and Zaret 2014). Recruitment of chromatin remodeling enzymes by pioneer factors is a critical step in creating accessible DNA (King and Klose 2017). The Snf2 family of chromatin remodelers use energy from ATP hydrolysis to exchange, evict, and reposition nucleosomes within chromatin (Clapier and Cairns 2009). To date, most studies of pioneer factors have focused on their role as master regulators of cellular differentiation and lineage reprogramming

Corresponding author: christopher.baker@jax.org Article published online ahead of print. Article and publication date are online at http://www.genesdev.org/cgi/doi/10.1101/gad.333542.119.
(Soufi et al. 2012; Zaret and Mango 2016), leaving open their necessity in other cellular processes requiring access to DNA such as meiotic recombination.

Meiosis, which is essential for all sexual reproduction, involves major reorganization of chromatin, along with chromosome condensation, pairing, and, ultimately, genetic recombination. The outcome of recombination is the exchange of genetic material between parental chromosomes, resulting in the shuffling of alleles between generations. In mammals, fungi, and plants, recombination is concentrated at specialized sites termed hot spots (Baudat et al. 2013; Tock and Henderson 2018). The physical exchange of DNA is initiated when the topoisomerase SPO11 is recruited to hot spots, where it induces the double-strand breaks (DSBs) required for DNA exchange between homologous chromosomes (Keeney 2001). Significant chromatin remodeling is likely a prerequisite to overcome chromatin barriers at hot spots and allow the proper targeting of SPO11.

In most mammals, hot spot locations are determined by the DNA-binding protein PRDM9 (Baudat et al. 2010;

(C) 2020 Spruce et al. This article is distributed exclusively by Cold Spring Harbor Laboratory Press for the first six months after the full-issue publication date (see http://genesdev.cshlp.org/site/misc/terms.xhtml). After six months, it is available under a Creative Commons License (Attribution-NonCommercial 4.0 International), as described at http://creativecommons.org/licenses/by-nc/4.0/. 
Myers et al. 2010; Parvanov et al. 2010). PRDM9 contains a SET domain, which catalyzes both histone H3 lysine 4 trimethylation (H3K4me3) and H3K36me3 (Buard et al. 2009; Eram et al. 2014), and a series of tandem C-terminal zinc finger domains that bind DNA. Prdm9 is highly polymorphic with the majority of differences in regions encoding the zinc finger domains (Berg et al. 2011; Kono et al. 2014). Allelic variants bind different DNA sequences, ultimately dictating the location of hot spots. PRDM9 binding occurs within nucleosome-depleted regions (NDRs) that are subsequently targeted for DSBs by SPO11 (Baker et al. 2014; Lange et al. 2016). Loss of Prdm9 results in the persistence of unrepaired DSBs, incomplete synapsis of homologous chromosomes, and pachytene arrest in meiotic prophase I (Hayashi et al. 2005; Sun et al. 2015). Interestingly, meiosis-specific loss of two Snf2 family chromatin remodeling factors, Hells (Lsh) and Ino80, each results in phenotypes similar to loss of Prdm9 (Zeng et al. 2011; Serber et al. 2016), indicating that these chromatin remodelers might play direct roles in nucleosome remodeling at recombination hot spots.

Given the broad similarity in requirements to create accessible DNA for both pioneer factor-mediated control of gene regulation and meiotic recombination, we postulated that PRDM9 functions as a pioneer factor to overcome chromatin barriers at hot spots by recruitment of chromatin remodeling enzymes.

\section{Results}

\section{Hot spots are marked by a unique epigenomic state}

To functionally annotate the chromatin landscape of male germ cells, we determined co-occupancy of histone modifications using ChromHMM (Ernst and Kellis 2012).
ChromHMM uses a hidden Markov model to classify genomic regions into "states" by identifying combinatorial patterns of chromatin features, in this case histone modifications. Using ChromHMM, we solved an 11-state model using new and our previously published nucleosome-resolution ChIP-seq data of 10 histone modifications or histone variants (Fig. 1A, Supplemental Table 1). ChIP-seq was performed using enriched germ cells from 12- to 14-d postpartum (dpp) C57BL/6J (B6) mice. After birth, the first wave of meiosis occurs semisynchronously (Bellve et al. 1977), allowing enrichment of meiotic stages when PRDM9 is active (Ball et al. 2016). This germ cell model recapitulated many of the previously observed chromatin states found in other cell types, including promoters and insulators (states 1), enhancers (states 2-5), transcriptionally active regions (state 6), Polycomb repressive complex-repressed (state 8), and heterochromatin (states 9 and 10) (Fig. 1A,B).

Of these 11 chromatin states, only state 7 was enriched for PRDM9-binding sites; it is distinguished by a unique combination of histone modifications. In addition to $\mathrm{H} 3 \mathrm{~K} 4 \mathrm{me} 3$ and H3K36me3, state 7 is characterized by H3K4me1 and H3K9ac (Fig. 1A,B). In contrast to other acetylation marks often found at enhancers (i.e., $\mathrm{H} 3 \mathrm{~K} 27 \mathrm{ac}$ and $\mathrm{H} 2 \mathrm{BK} 120 \mathrm{ac})$, only $\mathrm{H} 3 \mathrm{~K} 9 \mathrm{ac}$ is enriched at hot spots. The majority $(78 \%, 14,773 / 18,900)$ of all state 7 locations overlap with previously reported locations of B6 meiosis-specific DSBs (Smagulova et al. 2016) and PRDM9-dependent H3K4me3 modification (Fig. 1C; Baker et al. 2014), highlighting that state 7 represents recombination hot spots. Interestingly, compared with other phyla (Choi et al. 2013; Yamada et al. 2018), we did not detect H2A.Z at hot spots in mice. Together, these data identify a unique combination of histone features that biochemically distinguish recombination hot spots from
A

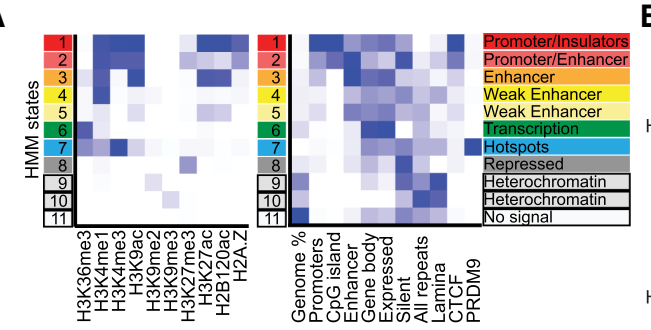

C

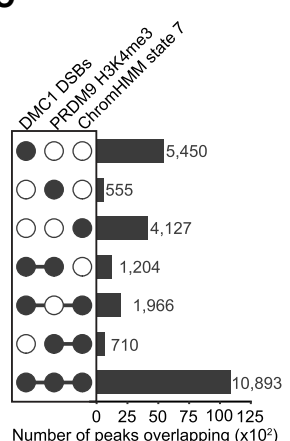

D

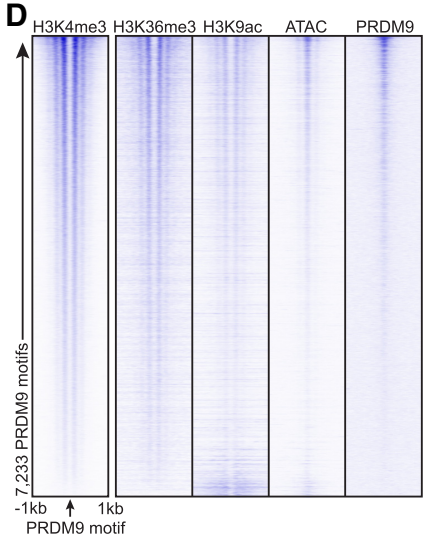

B

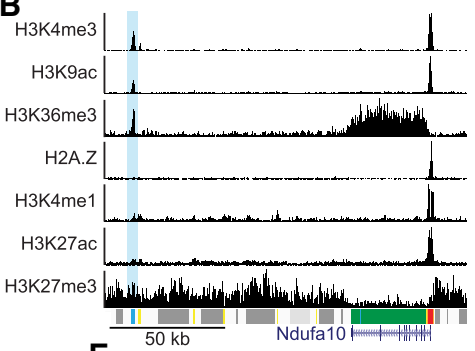

E

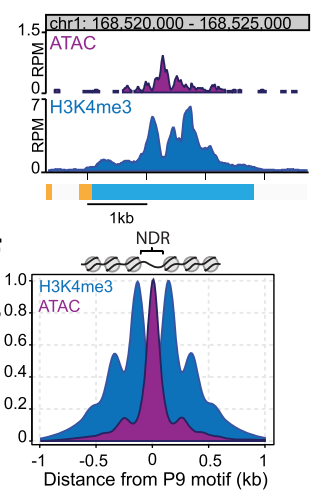

Figure 1. Recombination hot spots have a unique epigenomic signature and are sites of open chromatin. $(A$, left $)$ Heat map of the emission parameters for a 11-state ChromHMM model based on ChIP-seq for 10 histone modifications. (Right) Heat map showing fold enrichment for indicated annotations for each state. $(B)$ Example ChIP-seq profiles for data used to build ChromHMM model. (Bottom) HMM genome annotations. (Blue highlight) Example hot spot. (C) Upset plot showing relationships between meiotic DSBs (DMC1 ChIP), PRDM9-dependent H3K4me3, and HMM state 7. (D) Heat maps of histone modifications, ATAC-seq, and PRDM9 ChIP-seq. Heat maps are normalized counts per million (cpm) in 10-bp bins. (E) Genomic profile for example locus at $P b \times 1$ hot spot. $(F)$ Metaprofile of histone modifications and open chromatin at recombination hot spots from $D$ anchored by PRDM9 motif background subtracted and scaled to fraction of the maximum value. 
other regulatory elements and may be involved in creating chromatin accessibility for DSB formation.

\section{Hot spots are sites of accessible chromatin}

Given that recombination hot spots are marked by histone modifications associated with active chromatin, we measured DNA accessibility in germ cells using the assay for transposase-accessible chromatin (ATAC-seq) (Buenrostro et al. 2013). Biological replicates proved highly reproducible $(r=0.96)$, showed enrichment of open chromatin at promoters, and revealed typical nucleosome banding patterns (Supplemental Fig. 1A-D), all indicators of high-quality libraries. At PRDM9-dependent H3K4me3 sites, we detected increased DNA accessibility overlapping with PRDM9 motif locations (Fig. 1D-F). Overall, ATAC-seq identified fewer hot spots compared with H3K4me3 ChIP-seq $(2902$ ATAC peaks overlapping 13,498 PRDM9-dependent H3K4me3). Hot spots with greater PRDM9 binding and histone modification generally have more open chromatin (Fig. 1D). On average, the location of accessible DNA showed an inverse relationship to nucleosome positions at hot spots (Fig. 1E,F). Filtering ATAC-seq reads for those that fall within NDRs (i.e., $<120 \mathrm{bp}$ ) showed that open chromatin was highest where PRDM9 binds. Together, these data build on an earlier observation that detected increased DNase hypersensitivity at a single hot spot (Shenkar et al. 1991), and show that generally hot spots are sites of open chromatin.

\section{PRDM9 acts as a meiosis-specific pioneer factor to create open chromatin}

To determine whether the unique epigenomic state at hot spots is PRDM9-dependent, we used a strategy to maintain the same genetic background while changing Prdm9 alleles. To do so, we used a "knock-in" (KI) mouse strain that exchanges the zinc finger domain of the endogenous B6 Prdm9 $9^{\text {Dom2 }}$ allele with the zinc finger domain from CAST/EiJ mice (Prdm9 ${ }^{\text {Cst }}$ ) (Supplemental Fig. 1A; Baker et al. 2014). We measured chromatin accessibility and $\mathrm{H} 3 \mathrm{~K} 9 \mathrm{ac}$ in enriched KI germ cells (Supplemental Fig. 1E,F), combined with H3K4me3 (Baker et al. 2014), and compared these with the B6 results (Fig. 2A,B). Quantifying differences between B6 and KI identified 5775 ATAC peaks and $14,249 \mathrm{H} 3 \mathrm{~K} 9 \mathrm{ac}$ peaks increased in $\mathrm{KI}$ $($ FDR $<0.01)$. Of these, $92 \%$ of the ATAC peaks $\mid n=$ $5305)$ and $94 \%$ of the H3K9ac peaks $(n=13,408)$ overlap with reported PRDM9 ${ }^{\mathrm{Cst}}$-dependent $\mathrm{H} 3 \mathrm{~K} 4 \mathrm{me} 3$ peaks (Baker et al. 2014). Reciprocally, 1756 ATAC peaks and 7049 $\mathrm{H} 3 \mathrm{~K} 9 \mathrm{ac}$ peaks were increased in B6 compared with KI, with $94 \%$ of the ATAC peaks $(n=1643)$ and $91 \%$ of the H3K9ac peaks $(n=6431)$ overlapping known PRDM9 ${ }^{\text {Dom2 }}$-dependent $\mathrm{H} 3 \mathrm{~K} 4 \mathrm{me} 3$ peaks. Similar to the case with B6, open chromatin at hot spots in the KI strain is highest at PRDM9-binding sites (Supplemental Fig. 1H). These data show that hot spot chromatin that is accessible in B6 is not accessible in the KI. Instead, in the KI there are unique sites of accessible chromatin that overlap with known $\operatorname{Prdm} 9^{C s t}$ hot spots, sites that are not open in B6.
Next, if PRDM9 binding facilitated open chromatin, we reasoned that variants that disrupt PRDM9 binding should change DNA accessibility. B6 and DBA/2J (D2) strains share the $\operatorname{Prdm} 9^{\text {Dom2 }}$ allele, thus providing a natural experiment to determine the effect of genetic variation on chromatin accessibility. Recently we mapped quantitative trait loci (QTL) that regulate $\mathrm{H} 3 \mathrm{~K} 4 \mathrm{me} 3 \mathrm{lev}$ els in a genetic reference population whose genomes are homozygous mosaics of B6 and D2 (Baker et al. 2019). That study identified 1331 hot spots where the $\mathrm{H} 3 \mathrm{~K} 4 \mathrm{me} 3$ level is regulated by local variation (localQTL). We performed ATAC-seq on D2 mice and compared H3K4me3 levels and open chromatin at local-QTL to that from B6 (Fig. 2C). Hot spots with biased H3K4me3 levels also had biased open chromatin corresponding to increased frequency of single-nucleotide variants near PRDM9-binding sites. At each hot spot, we separately calculated scores for the PRDM9 motif for both B6 and D2 sequences. In general, the strain with the highest motif scores had the highest chromatin accessibility (Fig. 2C, right).

The programmed DSBs in meiosis are repaired primarily using the homologous chromatid as a template. This enabled us to determine whether the unique epigenomic landscape at hot spots occurs on both chromatids or only on the initiating chromatid. We performed ATAC-seq and H3K9ac ChIP-seq on spermatocytes from (B6xCAST)F1 hybrids and compared them with our published PRDM9 and H3K4me3 ChIP-seq data (Baker et al. 2015a). At asymmetric hot spots-i.e., those sites where PRDM9 preferentially binds one parental chromatid (Baker et al. 2015a; Smagulova et al. 2016) -both acetylation and chromatin accessibility are largely restricted to the parental chromatid to which PRDM9 binds (Fig. 2D,E). These data show that open chromatin is concordant with PRDM9 binding. Interestingly, however, they also suggest that hot spot chromatin is not open on the homologous chromosome, implying another mechanism is required to facilitate DNA accessibility for repair.

\section{Formation of open chromatin at hot spots precedes meiotic DSBs}

In somatic cells, DSBs lead to nucleosome remodeling and increased chromatin accessibility (Price and D'Andrea 2013). To determine whether this is also true of meiotic DSBs or whether chromatin remodeling precedes DSB formation, we tested whether the epigenomic state at hot spots is dependent on DSB formation. We performed ATAC-seq and ChIP-seq for H3K9ac and H3K4me3 on spermatocytes collected from 12- to 14-dpp mice lacking SPO11 (Baudat et al. 2000). In the absence of SPO11, we found wild-type levels of open chromatin and H3K9ac that show no quantitative differences compared with heterozygous littermate controls (Fig. 2F-H). These data agree with previous observations showing that H3K4me3 and H3K36me3 modifications at hot spots are independent of and therefore precede SPO11 binding and subsequent DSB formation (Grey et al. 2017). 
A
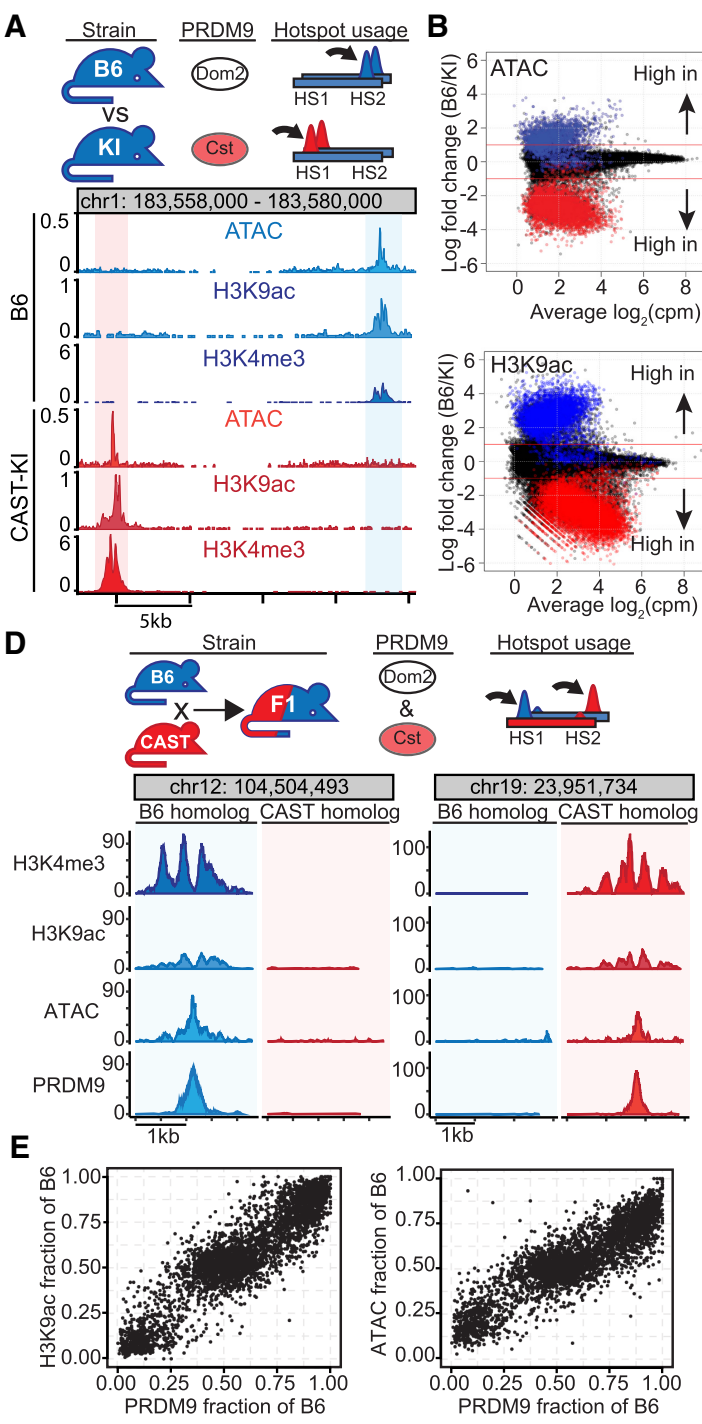

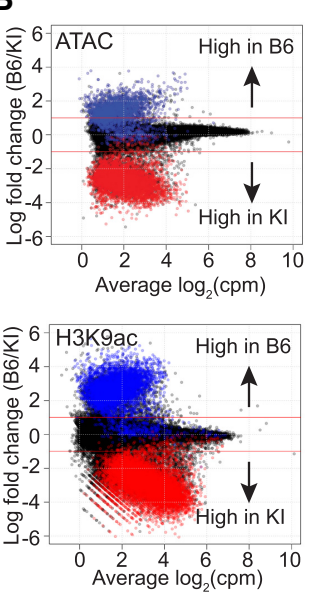

$\mathbf{F}$
C Strain PRDM9 Hotspot usage

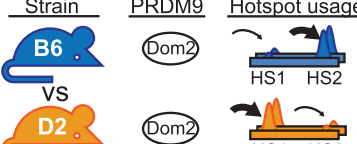

D2. एom2

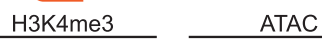

B6

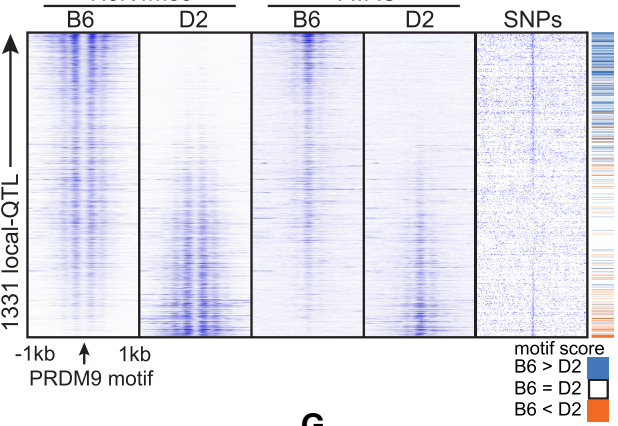

G
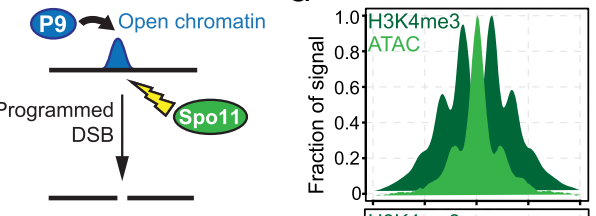
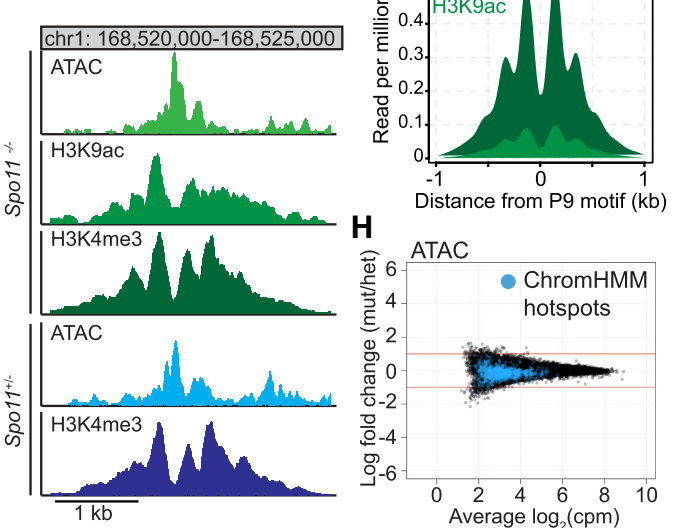

Figure 2. Open chromatin at hot spots is dependent on PRDM9 but not on DSBs. $(A$, top $)$ Graphic comparing B6 versus KI strains sharing the same genetic background and differing only in $\operatorname{Prdm} 9$ allele. Paired horizontal bars indicate homologous chromosomes, and height of the peaks suggests the level of open chromatin at the hot spot. (Bottom) Normalized (cpm) profiles of histone modifications and chromatin accessibility at two hot spots activated by different $\operatorname{Prdm} 9$ alleles. (B) MA plots comparing ATAC-seq (top) and H3K9ac (bottom) between B6 and KI. Prdm $9^{\text {Dom2 }}$ (blue) and $\operatorname{Prdm} 9^{C s t}$ (red) hot spot annotations are from Baker et al. (2014). (C, top) B6 and D2 strains represent different genetic backgrounds sharing the same $\operatorname{Prdm} 9^{\text {Dom2 }}$ allele. (Bottom) Heat maps showing H3K4me3, ATAC, single-nucleotide variant locations, and PRDM9 motif scores at 1331 local-QTL. ( $D$, top) Graphic outlining genetic makeup of heterozygous (B6xCAST)F1 hybrids with two Prdm9 alleles. (Bottom) Allele-specific profiles of H3K4me3, H3K9ac, ATAC, and PRDM9 (reads uniquely mapped to either the B6 or CAST haplotype). (E) Scatter plots of haplotype-specific PRDM9 binding $(n=3786)$ versus H3K9ac (left) or ATAC (right). (F, top) Open chromatin at hot spots occurs before programmed DSBs. (Bottom) H3K4me3, H3K9ac, and ATAC profiles in Spo11 ${ }^{-1-}$ mutant and H3K4me3 and ATAC profiles in heterozygous control at a representative hot spot (Pbx1). (G) Metaprofile of H3K4me3 and ATAC (top) and H3K4me3 and H3K9ac (bottom) at recombination hot spots from Figure 1D from Spo11 ${ }^{--}$. (H) MA plot of ATAC-seq from Spo11 ${ }^{-/-}($mut; $n=2)$ and Spo11 ${ }^{+-}($het; $n=1)$.

\section{PRDM9 overcomes chromatin barriers to transiently bind DNA}

Pioneer factors recognize partial DNA-binding motifs within nucleosomes (Soufi et al. 2012). Based on DNA sequence, $\mathrm{PRDM} 9^{\mathrm{Dom} 2}$-binding sites (Smagulova et al. 2011) and PRDM9 ${ }^{\text {Cst }}$-binding sites (Supplemental Fig. S2A) are predicted to be occupied by nucleosomes. To measure nu- cleosome occupancy in the absence of PRDM9 binding in vivo, we combined data from a total of eight MNaseseq libraries from four Mus musculus domesticus mouse strains (B6, D2, [B6xD2]F1, and WSB/EiJ) that do not carry the $\operatorname{Prdm} 9^{\text {Cst }}$ allele, resulting in $>328$ million reads. Hot spots were sorted based on MNase signal strength across the central 100-bp overlapping PRDM9 ${ }^{\text {Cst }}$-binding sites (Supplemental Fig. S2B). We found that $\sim 50 \%$ of 
PRDM9 ${ }^{\text {Cst }}$-binding sites have nucleosome signals at the PRDM9-binding site and that these nucleosomes are remodeled in spermatocytes that express PRDM9 ${ }^{\text {Cst }}$ to become nucleosome-depleted regions. These data are in agreement with a recent study (Hinch et al. 2019) that found higher levels of nucleosome occupancy at PRDM9-binding sites in the absence of PRDM9. In addition to exploring PRDM9 in vivo, expression of PRDM9 ex vivo recapitulates aspects of hot spot activation, including allele-specific H3K4me3 deposition (Baker et al. 2015b). To further investigate whether PRDM9 binds nucleosomal DNA in an orthologous system, we aggregated MNase-seq signal from HEK293 cells expressing different PRDM9 alleles (Supplemental Fig. S2C; Baker et al. 2015b). HEK293 cells that either do not express PRDM9 or are transfected with the $P R D M 9^{A}$ allele have no obvious nucleosome pattern at PRDM9 ${ }^{\mathrm{C}}$ hot spots. In contrast, these same genomic regions have depleted nucleosomes at PRDM9 ${ }^{C}$-binding sites upon expression of $P R D M 9^{C}$ (Supplemental Fig. S2C). These observations support the germ cell data and suggest that nearly half of PRDM9-binding sites must be buried within nucleosomes.

Residence time of DNA-binding proteins can be estimated in vivo using nuclease protection assays such as ATAC-seq and DNAse hypersensitivity (Sung et al. 2014). Proteins with long residence times will protect DNA from nucleases, resulting in a characteristic footprint observed by decreased cutting frequency at binding sites. In vitro, PRDM9 has a long residency time when bound to synthetic DNA oligonucleotides (Striedner et al. 2017). Footprint analysis of ATAC-seq data from germ cells found that PRDM9 ${ }^{\text {Cst }}$ motif locations showed little evidence for nuclease protection (Supplemental Fig. 2D,E), relative to long-resident proteins like CTCF (Supplemental Fig. 2F,G), similar to other transiently binding pioneer factors (Swinstead et al. 2016). Together, these data suggest that PRDM9 ${ }^{\text {Cst }}$ can access target motifs even within nucleosomal DNA, and the lack of a clear footprint is consistent with PRDM9 having a short residency time in vivo.

\section{The chromatin remodeling factor HELLS is required for} proper synapsis

Our observations that hot spots are marked by histone modifications associated with active chromatin and show PRDM9-dependent formation of accessible chromatin all point to an unknown mechanism controlling chromatin remodeling. To identify candidate remodeling complexes, we reasoned that if chromatin remodeling is required for hot spot activation, loss of the remodeling enzyme would result in a phenotype similar to loss of Prdm9. Loss of either Ino80 (Serber et al. 2016) or Hells (Zeng et al. 2011) fulfills these criteria. Given Ino80 plays a major role in histone variant exchange, specifically H2A.Z and H2A (Papamichos-Chronakis et al. 2011), and we found that recombination hot spots in mice lack H2A.Z (Fig. 1A), we focused our attention on Hells.

To examine HELLS localization during spermatogenesis, we used immunohistochemistry to detect protein abundance in seminiferous tubules (Fig. 3A). HELLS showed high expression in both spermatogonia and meiotic cells, similar to a previous result (Zeng et al. 2011). HELLS expression overlaps with phosphorylated H2AX $(\gamma \mathrm{H} 2 \mathrm{AX})$, a marker of DSBs, with broad temporal colocalization in leptotene and zygotene stages. During pachytene, both HELLS and $\gamma \mathrm{H} 2 \mathrm{AX}$ are sequestered to the sex body (Fig. 3A, arrow). In addition, HELLS is coexpressed with PRDM9 in spermatocytes (Fig. 3A). The timing and colocalization of PRDM9 and HELLS are supported by recent single-cell transcript analysis (Jung et al. 2019) showing individual leptotene cells coexpress Hells and Prdm9 (Supplemental Fig. 3). Together, these data show that HELLS and PRDM9 are both present when recombination hot spots are activated.

Next, we used a conditional strategy to study the meiosis-specific function of HELLS. We used Stra8-iCre (Sadate-Ngatchou et al. 2008) to create male mice with a homozygous loss of Hells at the onset of meiosis (Hells CKO). Adult CKO mice had reduced testis weight (Fig. 3B) with extensive loss of postmeiotic germ cells (Fig. 3C), while testes from Hells heterozygous littermates (control) appear normal. This block in germ cell development recapitulates an earlier observation using allographs of testis tissue between $\mathrm{Hells}^{-/-}$mice and wild-type donors (Zeng et al. 2011). Cross sections of adult Hells CKO seminiferous tubules show loss of HELLS staining (Supplemental Fig. 4A). The loss of later stage germ cells correlates with a large increase in germ cell apoptosis in Hells CKO compared with heterozygous littermate controls (Fig. 3C,D). Finally, Hells CKO male mice did not sire any offspring when mated to normal B6 females. In summary, we confirm that HELLS is required for sperm production and fertility of male mice.

Using surface-spread meiotic chromatin in combination with immunohistochemistry, we found that HELLS is diffusely localized in leptotene and zygotene spermatocytes (Fig. 3F), similar to localization of PRDM9 in the same stages (Parvanov et al. 2017). Meiotic spreads showed that later in prophase I, after repair of the majority of autosomal DSBs, HELLS is restricted to the sex body. Staining for HELLS is absent from Hells CKO mice (Fig. $3 \mathrm{~F})$. To determine at which stage meiotic progression is blocked in Hells CKO mice, we staged spermatocytes using spreads stained for both $\gamma \mathrm{H} 2 \mathrm{AX}$ as a marker of DSBs and SYCP3 to characterize chromosome synapsis in adult male mice (Supplemental Fig. 4B). We found that Hells CKO mice have an increased proportion of spermatocytes in leptotene and zygotene stages and a nearly complete loss of normal pachytene and diplotene stages (Fig. 3F). Hells CKO cells scored as pachytene consistently show persistent $\gamma \mathrm{H} 2 \mathrm{AX}$ phosphorylation and incomplete synapsis absent from littermate controls (Supplemental Fig. 4B). These pachytene-like cells are negative for the testis-specific histone variant H1t (Cobb et al. 1999), a marker for late pachytene (Supplemental Fig. 4C). Overall, our data confirm that loss of Hells leads to meiotic arrest at the late zygotene to early pachytene stage (Zeng et al. 2011), with incomplete synapsis and persistent DSBs leading to spermatocyte apoptosis. 
$A_{\text {DAPI }}$

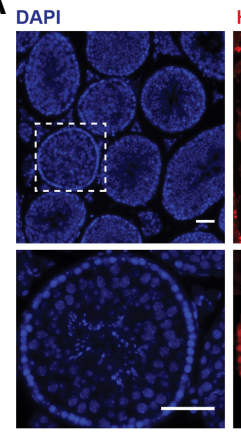

C

D

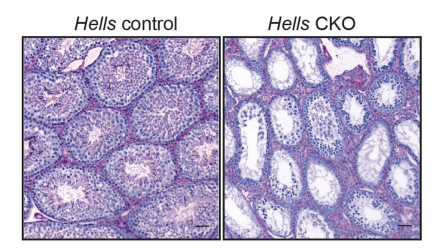

D

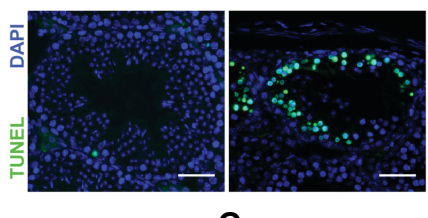

E

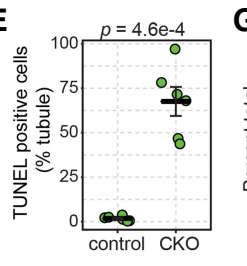

HELLS
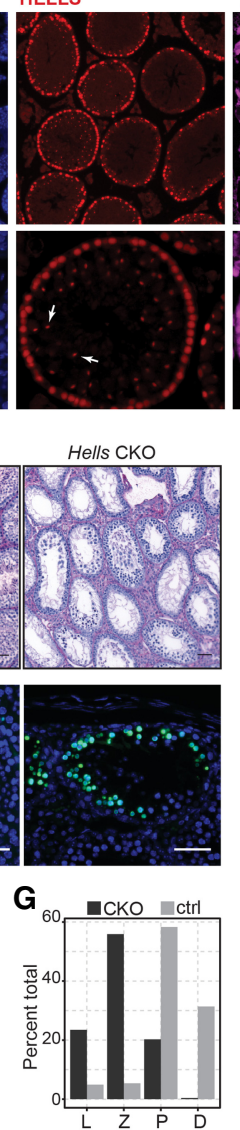
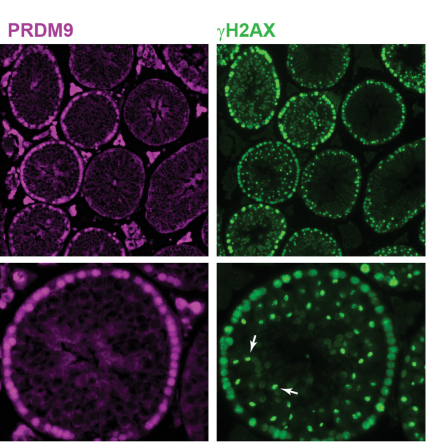

$\mathbf{F}$

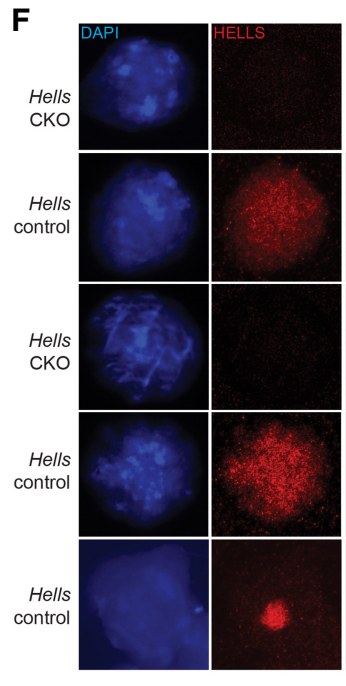

B
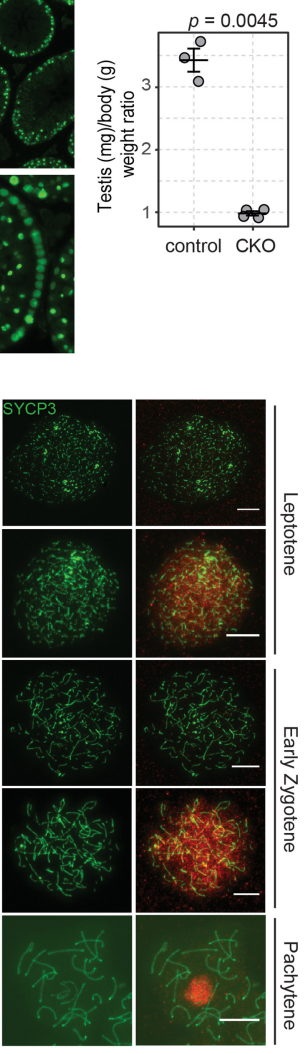

Figure 3. HELLS colocalizes with PRDM9 and is required for meiosis and spermatogenesis. (A) B6 adult testis cross sections immunolabeled with anti-HELLS (red), anti-PRDM9 (magenta), and anti- $\gamma \mathrm{H} 2 \mathrm{AX}$ (green). Nuclei were counterstained with DAPI in blue. (Arrow) Sex body. (B) Testis weight compared with body weight in adult Hells control and CKO mice (mean $\pm \mathrm{SEM}$; $P$-value was from Welch's two-sided test). (C) Adult testis cross sections stained with PAS. (D) TUNEL staining (green) of cross sections of adult testes from Hells control and CKO. Nuclei were counterstained with DAPI in blue. $(A, C, D)$ Scale bar, $50 \mu \mathrm{m}$. (E) Percentage of TUNEL-positive cells per tubule (mean \pm SEM; $P$ value was from Welch's two-sided test). (F) Chromatin spreads of spermatocytes from Hells control and CKO animals stained for DAPI (blue) and immunolabeled with anti-HELLS (red) and anti-SYCP3 (green). Scale bar, $10 \mu \mathrm{m}$. $(G)$ Quantification of meiotic prophase I stages, based on SYCP3 and $\gamma \mathrm{H} 2 \mathrm{AX}$ staining, in control and CKO mice $(n=200$ cells from two mice per genotype).
In Hells-null mice, meiotic DSBs are targeted to other functional elements

Because loss of Hells leads to incomplete synapsis and persistent $\gamma \mathrm{H} 2 \mathrm{AX}$, we next determined the levels and locations of meiotic DSB in mutant spermatocytes. To characterize DSBs cytologically, we used spread meiotic chromatin labeled with SYCP3 and DMC1. DMC1 is a meiosis-specific recombinase that binds to single-stranded DNA at the sites of DSBs (Neale and Keeney 2006). While zygotene spermatocytes from Hells CKO overall had similar levels of DMC1 foci as littermate controls (Fig. 4A), these foci persist in Hells CKO pachytene-like cells on synapsed chromosomes (Fig. 4B), indicating incomplete DSB repair.

While the number of DSBs were similar between Hells CKO and control, their genomic locations were dramatically different. To determine locations of meiotic DSBs, we identified genomic regions bound by DMC1 (Khil et al. 2012) in testes from adult CKO and littermate controls. In total, we identified 13,643 DMC1 peaks in Hells CKO and 15,718 in heterozygous littermates. Overall, Hells CKO germ cells lost DSBs at canonical hot spots and gained DSBs at other sites (Fig. 4C). For example, loss of Hells resulted in a 12-fold increase in DSBs at promoters and a concomitant decrease in DSBs at distal sites (Fig. 4D). Nearly all DMC1 peaks identified in Hells CKO germ cells overlap with the location of DSBs in $\operatorname{Prdm} 9^{-/-}(93.7 \%, 13,206 / 13,643)$, while only $15.7 \%(2475 / 15,718)$ of DMC1 sites identified in control germ cells overlap $\operatorname{Prdm} 9^{-/-} \mathrm{DMC} 1$ peaks (Fig. 4E).

To determine the epigenomic landscape targeted for DSBs in Hells CKO, we calculated enrichment of DMC1 sites and open chromatin within the ChromHMM states (Fig. 4F). While hot spots (state 7) were enriched for DSBs in the Hells control, in both Hells and Prdm9 mutants, DSBs were enriched in states 1 and 2, which are annotated as promoters, insulators, and enhancers. Interestingly, although $\mathrm{H} 3 \mathrm{~K} 4 \mathrm{me} 1, \mathrm{H} 3 \mathrm{~K} 4 \mathrm{me} 3$, and H3K9ac are present in states 1 and 2 (Fig. 1A), state 3 had a combination of histone modifications similar to classically defined enhancers that lack H3K4me3, and was less enriched for DSBs. States 1 and 2 were enriched for PRDM9-independent accessible chromatin; whereas state 7 only had PRDM9-dependent accessible chromatin in B6 compared with KI (Fig. 4F). Notably, unlike hot spots, states 1 and 2 were not enriched for H3K36me3. Furthermore, in Hells CKO, in which DSBs largely occur at state 1 and 2 locations, spermatocytes undergo apoptosis similar to loss of Prdm9. Together, these data suggest that in the absence of Hells, DSBs are retargeted to regions of open chromatin that lack the proper epigenome to become competent hot spots. 


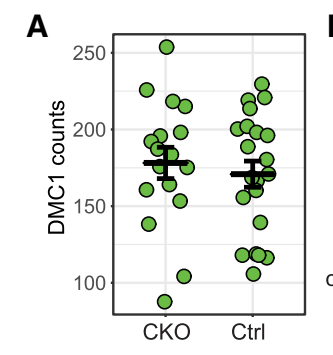

C

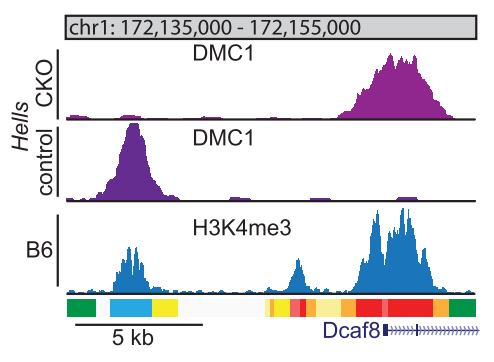

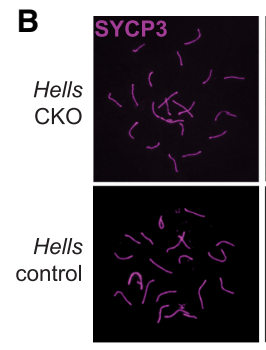

D

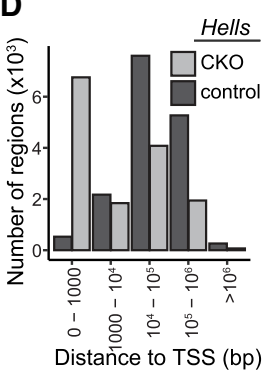

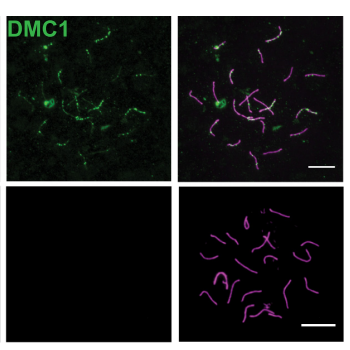

E

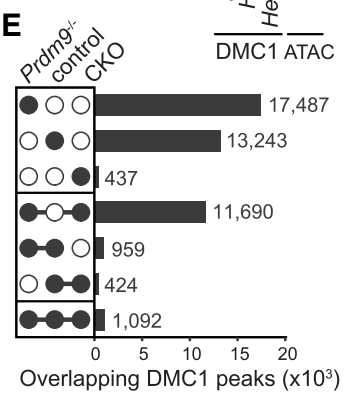

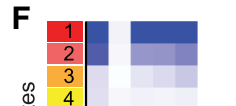
ments. (A) Quantification of DMC1 foci from chromatin spreads at zygotene/early pachytene stage spermatocytes in Hells $\mathrm{CKO}$ and control (Ctrl) animals (mean \pm SEM). (B) Representative chromatin spreads of a pachytene-like spermatocyte from Hells $\mathrm{CKO}$ and pachytene spermatocyte from Hells control. Spreads were immunolabeled with anti-SYCP3 (magenta) and anti-DMC1 (green). Scale bar, $10 \mu \mathrm{m} .(C)$ Profiles of DMC1 signal and $\mathrm{H} 3 \mathrm{~K} 4 \mathrm{me} 3$ level for representative locus in Hells CKO and control spermatocytes. ChromHMM state annotations are shown below profile tracks. $(D)$ Distribution of DMC1 ssDNA regions relative to transcription start sites (TSSs). (E) Intersection of DMC1 locations identified in spermatocytes from $\mathrm{Prdm} 9^{-/-}$and Hells control and CKO mice. $(F)$ Heat map showing fold enrichment of DMC1 locations and ATAC in ChromHMM states from Figure 1A.
HELLS is required for epigenomic activation of recombination hot spots

Because DSBs were retargeted in Hells CKO germ cells, we next examined the epigenome of the mutant spermatocytes. We first confirmed loss of HELLS in 12-dpp whole testes (Fig. 5A), when PRDM9 is active but prior to the Hells CKO meiotic arrest. ChIP-seq for H3K4me3 and ATAC-seq on enriched germ cell populations from 12-dpp male mice identified a significant reduction of epigenomic modification in Hells $\mathrm{CKO}$ mice at recombination hot spots (Fig. 5B,C; Supplemental Fig. 5). While 11,812 H3K4me3-modified sites are significantly reduced in Hells CKO compared with control ( $\log _{2}$ fold change < -1 , FDR $<0.01$ ), only 73 sites showed higher modification in Hells CKO. Annotating peaks using state 7, we found that both H3K4me3 $(n=11,125)$ (Fig. 5D) and chromatin accessibility $(n=1851)$ (Fig. $5 \mathrm{E})$ sites at hot spots were re- duced in Hells CKO. In contrast, among peaks overlapping HMM state 1 ( $n=31,161$ H3K4me3 peaks), there were virtually no sites that changed. Histological sections of 12-dpp seminiferous tubules from both Hells CKO and heterozygous control littermates showed similar stages in development (Supplemental Fig. S6A). Although measuring PRDM9 in whole testes from CKO mice by Western blot detected a modest reduction in protein abundance (Supplemental Fig. 6B,C), PRDM9 was readily detected in individual leptotene and zygotene spermatocytes (Supplemental Fig. 6D). These data show that HELLS is required for establishment of the epigenomic state and chromatin accessibility at hot spots.

\section{HELLS forms a complex with PRDM9}

To develop a model for temporal molecular characterization of hot spot activation, we chose a human cell line
A

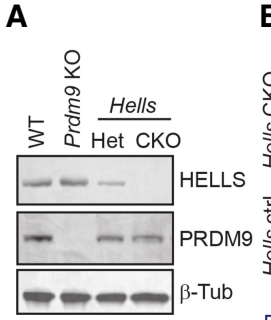

B
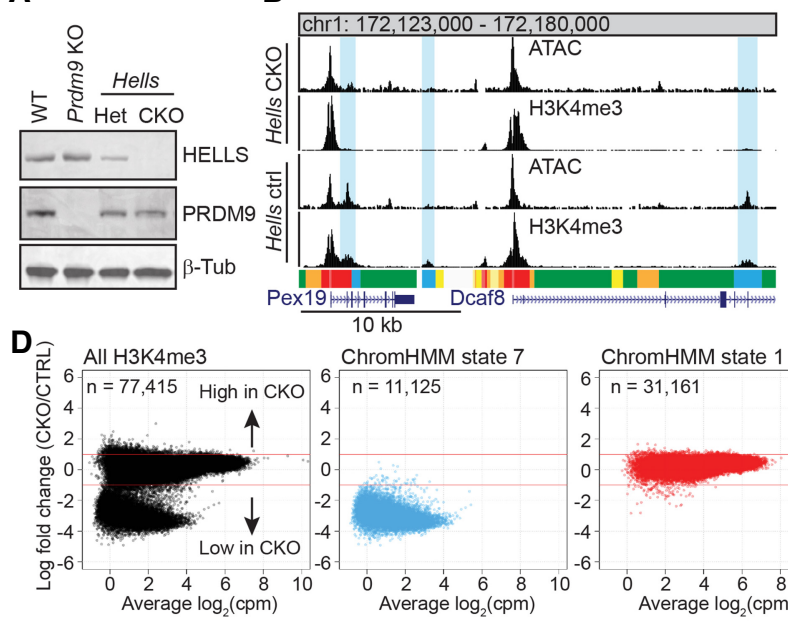

ChromHMM state 7

6
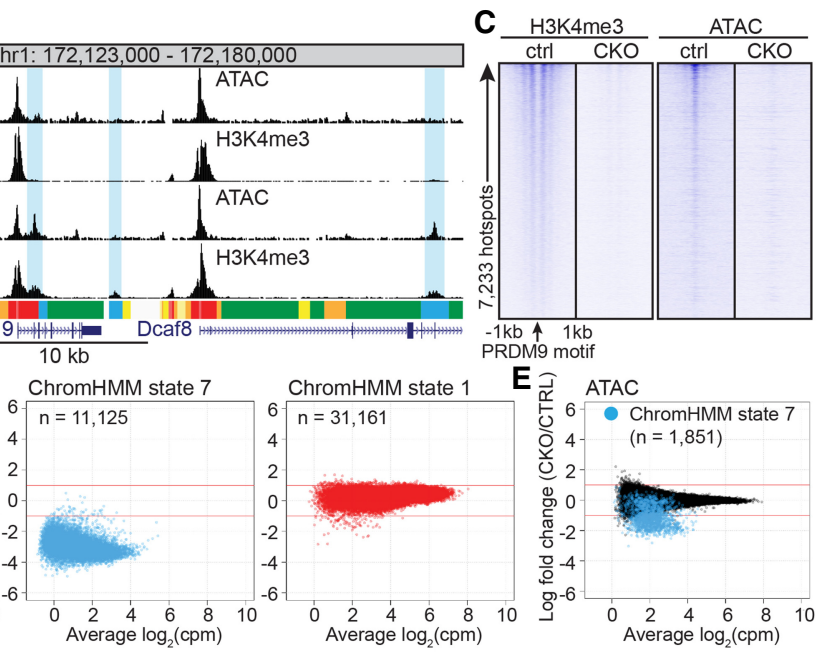

Figure 5. HELLS is required for histone modification and chromatin accessibility specifically at hot spots. (A) Western blot of whole-testis protein extract from B6 (WT), Prdm $9^{-/-}(\mathrm{KO})$, and Hells heterozygous (Het) and $\mathrm{CKO}(\beta$-Tubulin was used as a loading control). (B) Profile of ATAC and H3K4me3 levels from 12-dpp Hells CKO and control spermatocytes. HMM annotations are below. $(C)$ Heat map of $\mathrm{H} 3 \mathrm{~K} 4 \mathrm{me} 3$ and ATAC signal at all hot spots from Figure 1D (cpm in 10-bp bins). (D) MA plots of H3K4me3 levels comparing Hells $\mathrm{CKO}$ ( $n=$ $3)$ and homozygous controls $(n=3)$ for all H3K4me3 sites (left), HMM state 7 (middle; filtered for overlap with DSBs and PRDM9dependent $\mathrm{H} 3 \mathrm{~K} 4 \mathrm{me} 3)$, and HMM state 1 (right). (E) MA plot of ATAC signal (blue indicates HMM state 7; $n=2$ control; $n=3$ $\mathrm{CKO}$. 
that expresses HELLS and created a stably integrated Flag$P R D M 9^{C}$ allele under inducible control of the tetracycline promoter (HEK293-P9 ${ }^{\mathrm{C}}$ ) (Fig. 6A). Addition of doxycycline

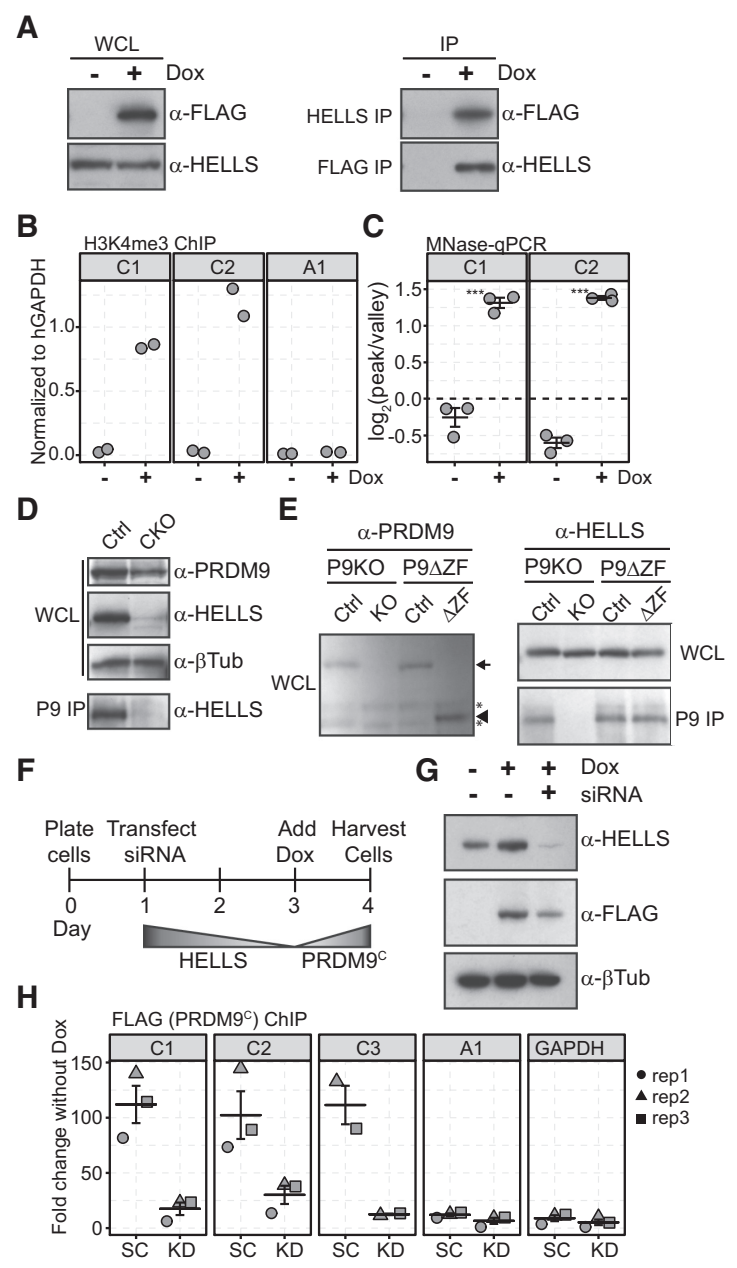

Figure 6. HELLS and PRDM9 form a complex in vivo. $(A$, left $)$ Western blot of whole-cell protein lysate (WCL) from HEK293$\mathrm{P9}^{\mathrm{C}}$ cells without $(-)$ or with $(+)$ addition of doxycycline. (Right) Western blot of immunoprecipitation (IP) using indicated antibodies on the left and blotted using antibodies on the right. (B) qPCR following H3K4me3 ChIP from HEK293-P9 ${ }^{\mathrm{C}}$ cells. (C1) Chr 3, $144 \mathrm{Mb}$; (C2) Chr 3, $4.4 \mathrm{Mb}$ are PRDM9 ${ }^{\mathrm{C}}$ hot spots; (A1) $\mathrm{Chr} 3,55 \mathrm{Mb}$ are PRDM9 ${ }^{\mathrm{A}}$ hot spots. (C) qPCR of DNA following MNase digestion of chromatin from HEK293-P9C cells. Numbers represent ratio of nucleosome peak versus NDR. Mean $\pm \mathrm{SEM}$; $\left({ }^{* * *}\right) P$-values $<0.001$, Welch's two-sided test. $(D)$ Western blots of either WCL or PRDM9 IP using protein lysate prepared from testes of either Hells control or CKO age-matched littermates. (E) Western blots of WCL and P9 IP from $\operatorname{Prdm} 9^{-1-}$ (P9KO) and $\operatorname{Prdm} 9^{\Delta Z F}(\mathrm{P} 9 \Delta \mathrm{ZF})$ animals. Control (Ctrl) lysates were prepared from age-matched littermates. (Arrow) Full-length P9; (arrowhead) truncated P9; (asterisk) nonspecific. (F) Schematic of experimental design. $(G)$ Western blots for WCL from HEK293 cells after addition of Dox and siRNA for scrambled control $(-)$ or HELLS $(+)$. (H) qPCR of Flag ChIP DNA following experimental design from $F$. Primers designed to PRDM9 ${ }^{\mathrm{C}}$ hot spots $(\mathrm{C} 1-\mathrm{C} 3)$, $\mathrm{PRDM}^{\mathrm{A}}$ hot spot $(\mathrm{A} 1)$, or GAPDH promoter (mean $\pm \mathrm{SEM}$ ). (SC) Scrambled siRNA; (KD) HELLS siRNA. led to robust expression of Flag-PRDM9 ${ }^{\mathrm{C}}$ and increased H3K4me3 at PRDM9 ${ }^{\mathrm{C}}$ hot spots (Fig. 6A,B). PRDM9 ${ }^{\mathrm{C}}$ expression also increased MNase sensitivity at $\mathrm{PRDM}^{\mathrm{C}}$. binding sites compared with adjacent regions (Fig. 6C), indicating nucleosome remodeling. Reciprocal coimmunoprecipitation (co-IP) using antibodies directed to either HELLS or PRDM9 (Flag) identified a doxycycline-dependent interaction between the two proteins (Fig. 6A right). We confirmed PRDM9-HELLS interaction in vivo by performing co-IP on protein lysates prepared from 12-dpp mouse testes. We detected a HELLS-PRDM9 interaction in testes from $\mathrm{B} 6$ mice and littermate controls but not in Hells CKO (Fig. 6D) or Prdm $9^{-/-}$(Fig. 6E). To test whether PRDM9-HELLS interaction requires assembly on chromatin, co-IP was repeated using a mouse strain expressing a PRDM9 variant that lacks the DNA-binding domain (PRDM9 ${ }^{\triangle \mathrm{ZF}}$ ) (Parvanov et al. 2017). We found that HELLS did interact with PRDM9 ${ }^{\triangle \mathrm{ZF}}$ (Fig. $6 \mathrm{E}$ ), suggesting that this interaction is independent of PRDM9-directed DNA binding.

We next showed that HELLS is required for robust PRDM9 binding at hot spots (Fig. 6F-H). HELLS expression in HEK293-P9 ${ }^{\mathrm{C}}$ cells was reduced using small interfering RNA (siRNA), followed by induced expression of PRDM9 $^{\mathrm{C}}$ (Fig. 6G). ChIP for Flag-PRDM9 ${ }^{\mathrm{C}}$ in HEK293$\mathrm{P9}^{\mathrm{C}}$ cells successfully detected robust binding at C-dependent hot spots and no binding at a PRDM9 ${ }^{\mathrm{A}}$ hot spot or at the GAPDH promoter (Fig. $6 \mathrm{H}$ ). Critically, upon loss of HELLS, PRDM9 ${ }^{\mathrm{C}}$ binding at hot spots was reduced to background levels (Fig. $6 \mathrm{H}$ ). Together, these data show that PRDM9 and HELLS form a complex in vivo and, by extension, suggests that active chromatin remodeling is required for robust PRDM9 binding at hot spots.

\section{HELLS is recruited to recombination hot spots through PRDM9}

Our experiments indicate that HELLS and PRDM9 interact and open chromatin at hot spots. This suggests a model in which PRDM9 recruits HELLS to hot spots to facilitate nucleosome remodeling for DSBs. To confirm this, we first tested whether HELLS binding at hot spots requires the presence of PRDM9 using our HEK293-P9 ${ }^{\mathrm{C}}$ cells. In the absence of PRDM $9^{\mathrm{C}}$, ChIP for HELLS did not identify binding at any of the five genomic locations tested by qPCR. In contrast, upon expression of PRDM9 ${ }^{\mathrm{C}}$, we observed significantly increased binding of HELLS only at C-hot spots and no change at negative controls (Fig. 7A), suggesting HELLS binding at hot spots is PRDM9 dependent.

To test for HELLS binding of hot spots in vivo, we performed ChIP using enriched germ cells from 12-dpp KI mice. Biological replicates were highly correlated (Pearson's $r=0.91$ ) (Fig. 7B) and identified 2675 HELLS-binding sites. Visually, we detected clear HELLS ChIP signal at recombination hot spots (Fig. 7C) and promoters (Supplemental Fig. 7A,B). HELLS peaks were annotated based on overlap with PRDM9-dependent H3K4me3 peaks from KI mice (Baker et al. 2014) and TSSs representing promoters (Fig. 7D). The largest class of HELLS-binding sites 
A HELLS ChIP-qPCR

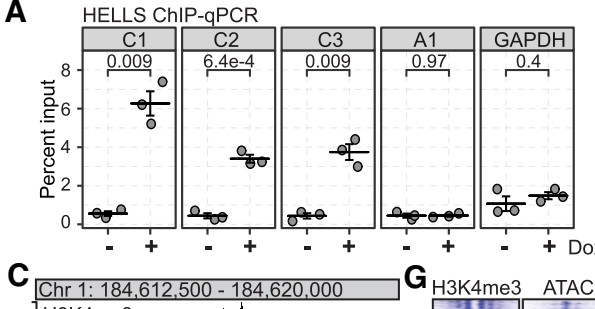

$\mathbf{G}_{\text {H3K4me3 ATAC HELLS IgG }}$
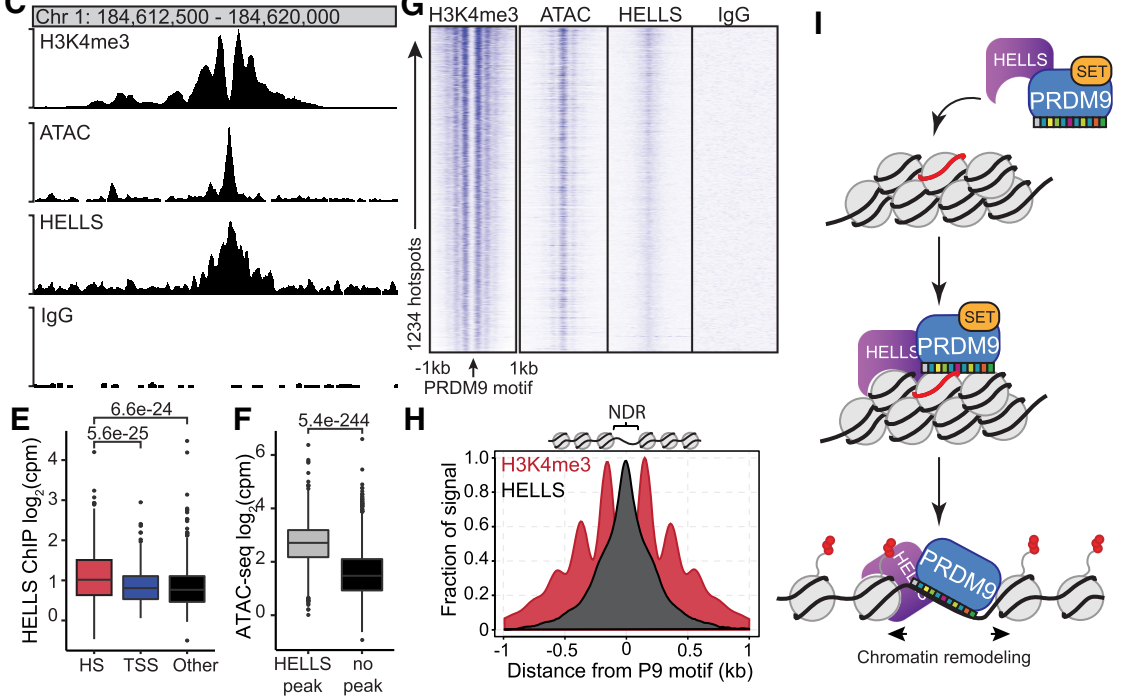

B

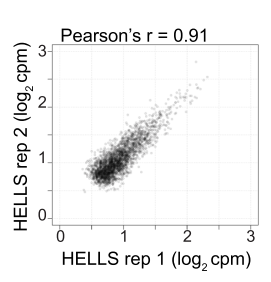

I

D

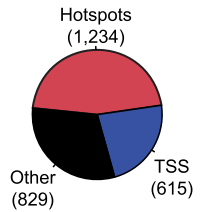

Other
$(829)$

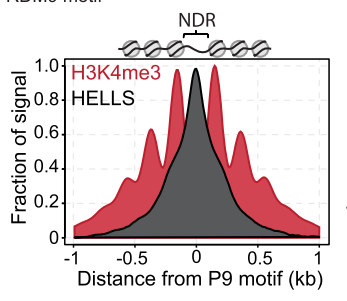
cols comparing distribution of HELL binding $(n=$ same as in $D ; P$-values from Welch's two-sided test). (HS) Hot spots (red); (TSS) promoters (blue); (other) unknown elements (black). (F) Box plot comparing distribution of open chromatin at hot spots with (gray; $n=1230$ ) or without (black; $n=4529$ ) a HELLS ChIP-seq peak $(P$-values were from Welch's two-sided test). (G) Heat maps of H3K4me3, ATAC, HELLS, and IgG signals $\pm 1 \mathrm{~kb}$ from PRDM $99^{\text {Cst }}$ motif. $(H)$ Metaprofile of H3K4me3 (red) and HELLS (gray) signals at hot spots from $G$ (all HELLS signals are from merged replicates). (I) Model for PRDM9-dependent recruitment to recombination hot spots. PRDM9 and HELLS bind before interacting with chromatin. Nucleosome remodeling creates open chromatin surrounding PRDM9binding sites.
( $n=1234,46.1 \%$ ) overlapped known PRDM9 ${ }^{\text {Cst }}$ hot spots. HELLS binding was stronger at hot spots compared with promoters $\left(p=5.6 \times 10^{-25}\right)$ or other unannotated regions $\left(p=6.6 \times 10^{-24}\right)$ (Fig. 7E). Of all HELLS binding regions, $97 \%(n=2591)$ fell within regions of open chromatin identified in KI germ cells. Moreover, hot spots with a HELLS ChIP-seq peak $(n=1230)$ had significantly higher levels of open chromatin $\left(p=1.3 \times 10^{-244}\right)$ compared with those without $(n=4529)$ (Fig. $7 F)$. In addition, genes with a HELLS peak at their promoters are generally expressed at significantly higher levels than those without (Supplemental Fig. 7C). Given that HELLS is a chromatin remodeling factor, HELLS might bind at hotpots via nucleosomes; however, the maximum average HELLS signal is found at the PRDM9-binding site (Fig. 7G,H). Together with the protein-protein interaction experiments, these results show that PRDM9 recruits HELLS to hot spots.

\section{Discussion}

Pioneer function is defined by three features: (1) the ability to recognize partial motifs within nucleosomal DNA, (2) acquisition of active histone modifications, and (3) increased chromatin accessibility. Here we show that together HELLS and PRDM9 fulfill these criteria at hot spots. PRDM9 brings two of the salient features of canonical pioneer factor-mediated chromatin reorganization into one molecule: (1) a sequence-specific targeting domain that provides locational specificity and can interact with nucleosomal DNA, and (2) the ability to catalyze epigenetic marks associated with active chromatin. Both of these features are required for meiotic recombination (Parvanov et al. 2017; Diagouraga et al. 2018). While the number of recombination hot spots are generally depleted from repressive regions of chromatin marked by H3K9me2/me3 (Walker et al. 2015; Patel et al. 2019), hot spots that are found in these domains show nearly similar levels of PRDM9-dependent histone modification as those found outside of $\mathrm{H} 3 \mathrm{~K} 9 \mathrm{me} 2 / 3$ domains, supporting the idea that even within heterochromatin, PRDM9 and HELLS have the capacity to create open chromatin. Additionally, a previous experiment characterizing the chromatin landscape using purified populations of male germ cells at stages earlier or later than leptotene/zygotene did not detect open chromatin at hot spots (Maezawa et al. 2018). This observation supports the role of a PRDM9-HELLS complex in creating de novo open chromatin at hot spots during a developmentally narrow window. Further evidence for a pioneer factor role for PRDM9 comes from our ex vivo analyses. Ectopic expression of PRDM9 in cells expressing HELLS recapitulates allelespecific histone modification and nucleosome remodeling. This suggests that the ability for PRDM9 to function as a pioneer factor is independent of chromatin structure that might be unique to meiosis.

The ability to target DSBs to open chromatin is critical for meiotic recombination. In mice, meiotic DSBs (Baker et al. 2014; Lange et al. 2016) and crossovers identified through single-sperm sequencing (Hinch et al. 2019) are concentrated within hot spot NDRs. In the absence of open chromatin at hot spots, by loss of either HELLS or 
PRDM9 (Brick et al. 2012), SPO11 preferentially targets other regions of accessible chromatin to create DSBs. Hot spots are marked by a unique combination of histone modifications not found at other regulatory elements, specifically H3K4me1, H3K4me3, H3K36me3, and H3K9ac. These observations support a precedent set by an early study that found enrichment of H3K9ac at an individual hot spot (Buard et al. 2009), and a recent survey of histone modifications in purified populations of male meiotic cells found that out of six histone acetylation antibodies tested, only H3K9ac showed high enrichment at hot spots (Lam et al. 2019). It is possible that this unique combination of histone modifications provides an epigenomic addressing system for directing placement or repair of DSBs through recruitment of an epigenetic reader. A plausible candidate for such a reader is ZCWPW1 (Jung et al. 2019; Li et al. 2019|, which is coexpressed with PRDM9 during the leptotene/zygotene phase and carries two protein domains that recognize H3K4me3 and H3K36me3. While the redirection of DSBs to other regulatory elements usually results in a meiotic block and infertility, notably, the severity of the infertility phenotype caused by loss of PRDM9 depends on the genetic background (Mihola et al. 2019), suggesting other components in action. Finally, through creation of aberrant sites of open chromatin, the pioneer function of the HELLS-PRDM9 complex could provide a molecular mechanism by which particular alleles are associated with genomic instability in certain cancers (Houle et al. 2018).

Here we identified that the chromatin remodeling enzyme HELLS is a second critical component of recombination initiation. Chromatin accessibility and histone modification at hot spots require HELLS, and furthermore, HELLS localizes at hot spots through interaction with PRDM9. HELLS is a member of the SNF2 family of ATP-dependent chromatin remodeling enzymes that use the energy of ATP to reposition, remodel, and remove histones from DNA substrate (Clapier and Cairns 2009). HELLS is misregulated in human tumors (von Eyss et al. 2012) and mutated in patients with immunodeficiency-centromeric instability-facial anomalies syndrome (Thijssen et al. 2015). Mice with systemic loss of HELLS exhibit prenatal lethality (Geiman et al. 2001); therefore, most functional studies of HELLS have required ex vivo cell culture. Notable exceptions are two studies showing that loss of HELLS in both male and female germ cells results in errors in synapsis and DSB repair during meiosis (De La Fuente et al. 2006; Zeng et al. 2011). Here, using conditional loss of function, we confirmed the requirement of HELLS in meiotic progression and further defined the mechanistic role of HELLS in chromatin remodeling at hot spots.

HELLS has been implicated in the establishment of genome-wide methylation patterns and chromatin repression (Yu et al. 2014; Termanis et al. 2016). HELLS ATPase domain is required for these functions (Ren et al. 2015; Termanis et al. 2016), and ATP is necessary for nucleosome remodeling in vitro (Jenness et al. 2018). If HELLS functioned to close chromatin in meiotic cells, we would have expected to see an increase in open chro- matin in CKO mice; instead, we detected a near universal closing of chromatin at hot spots upon loss of HELLS. Given that our CKO strategy ablated HELLS primarily in meiotic cells, these epigenomic experiments are not optimal for determining the consequences of loss of HELLS in other cell types but support the idea that the primary role of HELLS in meiosis is creating open chromatin at hot spots. In contrast, ChIP for HELLS was performed on a population of male germ cells, including spermatogonia. In addition to hot spots, we found that HELLS is enriched at promoters associated with higher gene expression, in agreement with previous results in fibroblasts (von Eyss et al. 2012). However, like many cell types, promoters are constitutively open during spermatogenesis (Maezawa et al. 2018), supporting the idea that HELLS role in opening chromatin in prophase I is specific to hot spots. Together these data show that, in addition to its wellcharacterized role in generating repressive chromatin, HELLS is associated with increased chromatin accessibility. These disparate functions likely depend on HELLS protein binding partners.

Our evidence suggest that HELLS is recruited to hot spots through PRDM9, rather than being recruited as a consequence of PRDM9-dependent epigenomic modification. The HELLS-PRDM9 interaction persists in cells with a mutated PRDM9 lacking zinc fingers, supporting the idea that their interaction is independent of DNA binding by PRDM9. In addition, on average the maximum HELLS enrichment is found in the center of NDR at the PRDM9-binding site, rather than at the flanking nucleosomes. These observations support a model in which HELLS is recruited by direct interaction with PRDM9, and agree with the previous observation that HELLS-mediated chromatin remodeling in vitro requires interaction with the zinc finger protein CDCA7 (Jenness et al. 2018).

Overall, data reported here give rise to a model (Fig. 7I) in which HELLS and PRDM9 form a meiosis-specific pioneer complex to create open chromatin at recombination hot spots. This model not only expands our knowledge about diversity in function of pioneer complexes in general but, more specifically, also poses interesting challenges with respect to meiotic mechanisms. Clearly further work is required to determine how the HELLS-PRDM9 complex is temporally regulated, how the epigenomic environment and open chromatin at hot spots recruits programmed DSBs, and how chromatin barriers are overcome on the template chromatid used to repair these breaks.

\section{Materials and methods}

Mice

C57BL/6J (B6, stock no. 000664), DBA/2J (D2, 000671), CAST/EiJ (CAST, 000928), B6.129X1-Spo11 ${ }^{\text {tm1Min }} / \mathrm{J}$ (Spo11 $\left.{ }^{-/-}, 019117\right), \mathrm{Tg}$ (Stra8-iCre)1Reb/J (Stra8-iCre, 008208), B6.Cg-Tg(ACTFLPe) 9205Dym/J (ATCFLPe, 005703), and B6;129P2-Prdm9 $9^{\text {tm1 1 Ymat }} / \mathrm{J}$ $\left(\operatorname{Prdm} 9^{K O}, 010719\right)$ are all available through The Jackson Laboratory. Mice carrying various Prdm9 alleles B6.Cg-Prdm $9^{\text {tm 1.1Kpgn } / ~}$ Kpgn (Baker et al. 2014) and B6.Cg-Prdm9 $9^{\text {tm3.1Kpgn }} /$ Kpgn 
$\left(\operatorname{Prdm} 9^{\Delta Z n f}\right)$ (Parvanov et al. 2017) were described previously. Frozen embryos of the EUCOMM allele C57BL/6NTac-

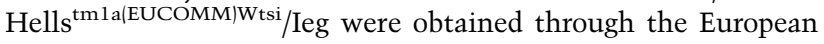
Mouse Mutant Archive (EMMA 04583) and implanted into pseudopregnant B6 females at The Jackson Laboratory. $H_{e l l s}{ }^{\text {tm1a(EUCOMM)Wtsi/+ }}$ pups were crossed to ATCFLPe to remove the FRT flanked neomycin selection cassette, and resulting mice were inbred to make the conditional ready $H_{e l l s}{ }^{\text {tmic(EUCOMM)Wtsi }}$ allele. These Hells conditionally ready mice are available through The Jackson Laboratory (B6.Cg-

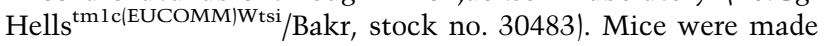
homozygous for a germ cell-specific loss of function in two steps by crossing $\mathrm{Hell}^{\mathrm{tm}^{1 c} \mathrm{c}}$ homozygotes to Stra8-Cre to create male mice with germ cells heterozygous for Hells ${ }^{+/ t m 1 d}$ and Stra8$\mathrm{Cre}$. These male mice were backcrossed to female Hells ${ }^{\text {tm1c }}$ homozygotes to produce Hells ${ }^{\text {tm1c/tm1d }}$; Stra8-Cre offspring, of which testes from male mice would lack functional Hells (Hells ${ }^{\text {tm1d }}$ homozygotes) and were used throughout this study as CKO. Fertility testing was performed on three Hells CKO male mice. Starting at $8 \mathrm{wk}$ of age, the male CKO mice were each mated to two or more confirmed B6 breeder females for at least $4 \mathrm{mo}$, and no pregnancy or litters were ever recorded. All animal experiments were approved by the animal care and use committee of The Jackson Laboratory (Animal Use Summary 16043).

\section{Cell culture and HEK293-P9 ${ }^{C}$ cells}

HEK293-P9C cells were created using the T-REx system (Thermo Fisher K102001) and the T-REx-293 (HEK293) cell line (Thermo Fisher R71007). Human Flag-tagged PRDM9 ${ }^{C}$ (Baker et al. 2015b) was subcloned into the pcDNA4/TO vector. T-REx-293 cells were transfected using Lipofectamine 3000 (Invitrogen), allowed to recover for $48 \mathrm{~h}$, and subsequently split and plated on $100-\mathrm{cm}$ plates maintained with $5 \mu \mathrm{g} / \mathrm{mL}$ blasticidin (Thermo Fisher R210-01) and $300 \mu \mathrm{g} / \mu \mathrm{L}$ zeocin (Thermo Fisher R250-01) to allow for clonal expansion. Individual colonies were picked, expanded, and tested by Western blot for expression of Flag$\mathrm{PRDM}^{\mathrm{C}}{ }^{\mathrm{C}}$. A single cell line was subsequently used for all studies. Flag-PRDM ${ }^{\mathrm{C}}$ expression was induced by replacing culture media with fresh media containing $1 \mu \mathrm{g} / \mathrm{mL}$ doxycycline (Sigma D9891) in DMSO (Sigma D2650-5 $5 \mathrm{~mL}$ ) and grown for an additional 24 h. Human Hells siRNA (Origene SR302088) knockdown was performed in HEK293-P9C ${ }^{C}$ cells with either $100 \mathrm{nM}$ siRNA or universal scrambled control (Origene SR30004) using Lipofectamine RNAiMAX (Thermo Fisher 13778030).

\section{Western blot, protein extraction, and immunoprecipitation}

Protein extracts for Western blots from cultured HEK293-P9C cells were prepared as described previously (Baker et al. 2015b). Protein extracts for co-IP from HEK293 cells was prepared using HEPES-based buffer as described previously (Baker et al. 2015b). For co-IP, protein lysates were diluted to $500 \mu \mathrm{L}$ using HEPES buffer and treated with $4 \mu \mathrm{L}$ antibody and $20 \mu \mathrm{L}$ of Protein-A/G magnetic beads (Fisher). Immunocomplexes were incubated overnight at $4^{\circ} \mathrm{C}$ with rotation, washed three times with HEPES wash buffer (50 mM Hepes-KOH at pH 7.4, $150 \mathrm{mM} \mathrm{NaCl}, 0.4 \%$ NP40 ), and eluted with $20 \mu \mathrm{L}$ of $2 \times$ sample loading buffer $(50 \mathrm{mM}$ Tris-HCL at $\mathrm{pH} 6.8,2 \%$ SDS, $10 \%$ glycerol, $100 \mathrm{mM}$ DTT, $0.05 \%$ bromophenol blue) heated for $10 \mathrm{~min}$ at $95^{\circ} \mathrm{C}$. Primary antibodies for Western blots and immunoprecipitation included anti-Flag M2 (Millipore/Sigma F3165), anti-PRDM9 (custom) (Parvanov et al. 2017), anti-HELLS (Millipore/Sigma ABD41, lot 3069868), and anti- $\beta$-Tubulin (Sigma T4026, lot 125M4884V). Secondary antibodies for Western blots included goat antimouse
HRP (Bio-Rad 170-6516), goat antirabbit HRP (Bio-Rad 172-1019), and donkey antiguinea pig (Millipore AP193P).

Histological sections, chromatin spreads, and immunofluorescence

Testes were fixed for 12-16 h with Bouin's solution and embedded in paraffin wax. Five-micrometer sections were prepared and stained with periodic acid Schiff diastase (PAS). Samples prepared for immunofluorescence were fixed in $4 \%$ PFA in PBS for $12-16 \mathrm{~h}$ as described previously (Parvanov et al. 2017). Prior to immunofluorescent staining, sections were deparaffinized with xylene washes and rehydrated through a gradient of ethanol washes $(95 \%, 70 \%$, and $50 \%, \mathrm{PBS})$ and then boiled in $10 \mathrm{mM}$ sodium citrate buffer (pH 6.0) for $10 \mathrm{~min}$. After being cooled and washed in PBS, the slides were blocked with $10 \%$ normal donkey serum (Jackson ImmunoResearch Laboratories 017-000$121), 3 \%$ BSA, $0.2 \times$ protease inhibitor cocktail, and $0.05 \%$ Triton-X 100 for $1 \mathrm{~h}$ at room temperature before adding primary antibodies and incubating overnight at $4^{\circ} \mathrm{C}$. Slides were washed three times for $5 \mathrm{~min}$ in PBS and incubated in secondary antibody and then washed three times for $10 \mathrm{~min}$ in PBS. Slides were treated with ProLong Gold antifade reagent with DAPI (Invitrogen P36935).

Chromosome spreads were prepared using the drying-down technique (Peters et al. 1997). For immunofluorescence, slides were blocked with $3 \%$ BSA, $1 \%$ normal donkey serum, and $0.005 \%$ Triton-X 100, and primary antibodies were incubated overnight. Slides were washed and treated with secondary antibodies as described above and treated with ProLong Gold antifade reagent with DAPI (Invitrogen P36935).

Primary antibodies used for immunofluorescence on cross sections and chromosome spreads included anti-PRDM9 (1:100) (Parvanov et al. 2017), anti-HELLS (1:250; Millipore/Sigma ABD41), anti- $\gamma \mathrm{H} 2 \mathrm{AX}$ (1:2000; Millipore 05636I, lot 2888552), anti-SYCP3 (1:500; Santa Cruz Biotechnology SC74569, lot J1314), anti-H1t (1:300; a gift from Mary Ann Handel), and antiDMC1 (1:200; Santa Cruz Biotechnology sc-8973). Secondary antibodies included goat antirabbit immunoglobulin $\mathrm{G}$ (IgG), Alexa fluor 594 conjugate (1:300; A-11037), goat antirabbit IgG, Alexa fluor 488 conjugate (1:500; ab150077), goat antimouse IgG, Alexa fluor 488 conjugate (ab150113), goat antimouse IgG, Alexa fluor 647 conjugate (1:300; A-21236), goat anti-guinea pig IgG, and Alexa fluor 488 conjugate (1:300; A-11073).

TUNEL assay was performed on testis sections from 6-wk-old Hells heterozygous control or CKO mice using the in situ cell death detection kit (Roche 11684795910) according to the manufacturer's protocol.

\section{Chromatin immunoprecipitation and ChIP-seq library preparation}

Isolation of enriched population of germ cells for ChIP for histone modifications and ATAC-seq was performed as described previously (Baker et al. 2014) on 12- to 14-dpp mice. Antibodies used for histone ChIP experiments were anti-H3K4mel (Millipore/ Sigma 07-436, lot 2289129), anti-H3K4me3 (Millipore/Sigma 07-473, lot 3018770), anti-H3K9ac (Active Motif 39137, lot 09811002), anti-H3K27ac (Abcam ab4729, lot GR211893-1), anti-H3K27me3 (Millipore/Sigma 07-449, lot 2475696), antiH2BK120ac (Active Motif 39120, lot 01008001), and antiH2A.Z (Millipore/Sigma 07-594, lot 2455725). For ChIP of H3K9ac, H3K27ac, and H2BK120ac used in the ChromHMM analysis, germ cell isolation and ChIP buffers were supplemented with $20 \mathrm{mM}$ sodium butyrate.

ChIP for Flag-PRDM9 was described previously (Baker et al. 2015a) and modified for HELLS to include a dual cross-linking 
step using disuccinimidyl glutarate (DSG) to first capture proteinto-protein interactions. Seminiferous tubules were placed in $5 \mathrm{~mL}$ of PBS (pH 7.4) supplemented with $1 \mathrm{mM} \mathrm{MgCl}_{2}$. A fresh $0.25 \mathrm{M}$ stock of DSG was prepared in DMSO, added at a final concentration of $2 \mathrm{mM}$, and incubated for $30 \mathrm{~min}$ at room temperature with rotation. After incubation, fresh paraformaldehyde was added to a final concentration of $1 \%$, and tubules were incubated for $5 \mathrm{~min}$ with rotation and quenched with $125 \mathrm{mM}$ glycine. The remaining procedure for ChIP was followed as described (Baker et al. 2015a) using anti-HELLS (Millipore/Sigma ABD41, lot 3069868) or normal mouse IgG (Millipore/Sigma 12-371, lot 2880788).

Single-strand DMC1 ChIP-seq was performed as described (Khil et al. 2012) using DMC1 antibody (Santa Cruz Biotechnology sc-8973, lot K2614) on testes from 6-wk-old male mice collected from two independent biological replicates of Hells CKO and heterozygous littermate controls.

ChIP libraries were prepared for high-throughput sequencing either using Bioo Scientific's NEXTflex ChIP-seq kit without size selection (for ChromHMM data) or the KAPA hyper kit (Kapa Biosystems) for all other libraries. Library quality and size distribution were visualized using a Bioanalyzer (Agilent). All samples were sequenced using either the Illumina HiSeq2500 or NextSeq platforms.

Quantitative PCR was performed using PowerUp SYBR Green $2 \times$ master mix (ThermoFisher) on a ViiA 7 real-time PCR system (Thermo Fisher). PCR was carried out for 40 cycles followed by melting curve analysis. All samples were run with triplicate technical reactions, and cycle number was determined using an automated threshold analysis. MNase sensitivity assay in HEK293$\mathrm{P9}^{\mathrm{C}}$ cells before and after doxycycline addition was performed as described for spermatocytes (Baker et al. 2014). Oligonucleotides used for qPCR in this study are listed in Supplemental Table 2.

\section{ATAC-seq sample preparation and sequencing}

For B6, KI, D2, and (BxC)F1 hybrid genotypes, ATAC-seq was performed on male germ cells using the original protocol (Buenrostro et al. 2013). ATAC-seq for Spo11 and Hells genotypes was performed using the modified FAST-ATAC protocol (Corces et al. 2016). For Hells genotypes, ATAC-seq libraries were prepared from two independent homozygous Hells wild-type $\left(\mathrm{Hell}^{\left.\mathrm{tm}^{\mathrm{m} 1 \mathrm{c}+}\right)}\right.$ and three independent Hells CKO (Hells ${ }^{\text {tm1c/s}}$; Stra8-iCre). Tn5 transposition was performed using $1 \times 10^{5}$ enriched male germ cell populations prepared from pooling testes from three to four individual mice similar to ChIP. ATAC-seq libraries were amplified using a total of eight to 10 amplification cycles and DNA purified using Agencourt AMPure XP beads (Beckman Coulter).

\section{Data analysis}

All reference sequence coordinates in this study correspond to UCSC mm10 (release 3/18/2013).

Chromatin segmentation was performed using ChromHMM as described (Ernst and Kellis 2012) solving for an 11-state solution on histone ChIP-seq libraries (Supplemental Table 1). Duplicate ChIP libraries were merged prior to ChromHMM processing. Fold enrichments were calculated using whole-testis CTCF occupancy and predicted cis-regulatory elements (Shen et al. 2012), lamina-associated domains from mouse embryonic stem cells (Meuleman et al. 2013), and in vitro PRDM9-binding sites (Walker et al. 2015). Gene expression levels from germ cells of agematched mice were downloaded from GEO GSE72833 (Ball et al. 2016). Expressed genes were defined by threshold transcripts per million (TPM) of more than three $(n=13,739)$. All remaining genes were annotated as "silent."
ATAC-seq fastq files were trimmed using trimmomatic (Bolger et al. 2014) using TRAILING:3 SLIDINGWINDOW:4:15 MINLEN:36 and removing Nextera adapters. For paired-end ATACseq samples, trimmed sequences were aligned using bowtie (version 0.12.9) (Langmead et al. 2009) with the following settings: -chunkmbs 2000 -S -X2000 -m1. Mapped reads were adjusted for Tn5 insertion site as described (Buenrostro et al. 2013). For single-end ATAC-seq, trimmed sequences were aligned using bwa mem (version 0.7.15) (Li and Durbin 2009) with default settings. Picard Tools was used to remove duplicates and calculate insert size metrics. ATAC-seq alignments were filtered to remove reads with $M A P Q<10$ and mitochondrial reads.

ChIP libraries were aligned using bwa mem with default settings (version 0.7.15) (Li and Durbin 2009). D2 ChIP-seq and ATAC-seq data were aligned to an in silico pseudogenome incorporating known variants (R78-REL1505) (Wu et al. 2010) and converted to $\mathrm{mm} 10$ reference coordinates using G2Gtools (accessible online at https://github.com/churchill-lab/g2gtools). Allele-specific ChIP analysis was performed using variant-aware alignment strategy EMASE (Raghupathy et al. 2018). To identify hot spots and ensure robust haplotype calls, peaks were selected that overlap with (BxC)F1 PRDM9 ChIP-seq summits and filtered for $>30$ reads that map uniquely to either B6 or CAST.

Both ChIP-seq and ATAC-seq peaks were called using MACS version 1.4.2 (Zhang et al. 2008) using a cutoff of $10^{-5}$. Duplicate reads were retained for ChIP against histone modifications and removing for HELLS ChIP. To build reference peakomes for each experiment, peaks were called on merged bam files from replicate experiments. Peak files across different strains or Prdm9 alleles were concatenated, sorted, and merged using bedtools (Quinlan and Hall 2010). Peaks overlapping ENCODE blacklist regions were removed for subsequent analysis. Read counts for each reference peakome were extracted from sample bam files using BedTools multicoverage and are available as processed data at NCBI GEO (GSE135896). Genomic profiles were visualized using the UCSC browser.

Statistical analysis was performed using $\mathrm{R}$ (release 3.4.1). For differential analysis, read counts were normalized using the trimmed mean of $M$-values method and differential analysis performed using edgeR (Robinson et al. 2010). Genome-wide false discovery rates (FDRs) were calculated by adjusting $P$-values following the Benjamini-Hochberg method. Annotation of peaks using genomic locus overlap was performed with bedtools intersect or HOMER mergePeaks functions (Heinz et al. 2013) and visualized using the UpSet package (Lex et al. 2014). Read matrices for heat maps were generated using the CoverageView R package after RPM normalization with 10-bp bins and visualized using Java TreeView (Saldanha 2004). ATAC-seq footprints were aggregated and normalized using ATACseqQC (Ou et al. 2018). Calculation of motif scores for predicted PRDM9-binding sites was performed by generating a position weighted matrix as outlined (Wasserman and Sandelin 2004).

\section{Acknowledgments}

We thank all members of the Baker laboratory for comments and discussion. We thank Petko Petkov and Anita Hawkins for $\operatorname{Prdm} 9^{\Delta Z n f}$ mice, as well as Tanmoy Bhattacharyya and Travis Kent for technical help with PRDM9 histological staining and staging seminiferous tubules. We thank Mary Ann Handel and Taneli Helenius for critical reading of the manuscript and suggestions. This work was supported by P01 GM099640 to K.P and G.W.C., and Jackson Laboratory start-up funds and R35 GM133724-01 to C.L.B., and was assisted by The Jackson 
Laboratory scientific services, which are supported through National Institutes of Health Cancer Core grant CA34196.

Author contributions: C.L.B. conceived the study. C.L.B. and G.W.C. were responsible for the methodology. C.L.B. and G.A. performed the formal analysis. C.S., S.D., N.B., H.T., and C.L.B. performed the investigation. K.P. was responsible for the resources. C.S. and C.L.B. wrote the original draft of the manuscript. K.P. and C.L.B. reviewed and edited the manuscript. K.P., G.W.C., and C.L.B. acquired the funding. C.L.B. supervised the study.

\section{References}

Baker CL, Walker M, Kajita S, Petkov PM, Paigen K. 2014. PRDM9 binding organizes hotspot nucleosomes and limits Holliday junction migration. Genome Res 24: 724-732. doi:10.1101/gr.170167.113

Baker CL, Kajita S, Walker M, Saxl RL, Raghupathy N, Choi K, Petkov PM, Paigen K. 2015a. PRDM9 Drives evolutionary erosion of hotspots in Mus musculus through haplotype-specific initiation of meiotic recombination. PLoS Genet 11: e1004916. doi:10.1371/journal.pgen.1004916

Baker CL, Petkova P, Walker M, Flachs P, Mihola O, Trachtulec Z, Petkov PM, Paigen K. 2015b. Multimer formation explains allelic suppression of PRDM9 recombination hotspots. PLoS Genet 11: e1005512. doi:10.1371/journal.pgen.1005512

Baker CL, Walker M, Arat S, Ananda G, Petkova P, Powers NR, Tian H, Spruce C, Ji B, Rausch D, et al. 2019. Tissue-specific trans regulation of the mouse epigenome. Genetics 211: 831-845. doi:10.1534/genetics.118.301697

Ball RL, Fujiwara Y, Sun F, Hu J, Hibbs MA, Handel MA, Carter GW. 2016. Regulatory complexity revealed by integrated cytological and RNA-seq analyses of meiotic substages in mouse spermatocytes. BMC Genomics 17: 628. doi:10.1186/s12864016-2865-1

Baudat F, Manova K, Yuen JP, Jasin M, Keeney S. 2000. Chromosome synapsis defects and sexually dimorphic meiotic progression in mice lacking Spo11. Mol Cell 6: 989-998. doi:10 .1016/S1097-2765(00)00098-8

Baudat F, Buard J, Grey C, Fledel-Alon A, Ober C, Przeworski M, Coop G, de Massy B. 2010. PRDM9 is a major determinant of meiotic recombination hotspots in humans and mice. Science 327: 836-840. doi:10.1126/science.1183439

Baudat F, Imai Y, de Massy B. 2013. Meiotic recombination in mammals: localization and regulation. Nat Rev Genet 14: 794-806. doi:10.1038/nrg3573

Bellve AR, Cavicchia JC, Millette CF, O'Brien DA, Bhatnagar YM, Dym M. 1977. Spermatogenic cells of the prepuberal mouse: isolation and morphological characterization. J Cell Biol 74: 68-85. doi:10.1083/jcb.74.1.68

Berg IL, Neumann R, Sarbajna S, Odenthal-Hesse L, Butler NJ, Jeffreys AJ. 2011. Variants of the protein PRDM9 differentially regulate a set of human meiotic recombination hotspots highly active in African populations. Proc Natl Acad Sci 108: 12378-12383. doi:10.1073/pnas.1109531108

Bolger AM, Lohse M, Usadel B. 2014. Trimmomatic: a flexible trimmer for Illumina sequence data. Bioinformatics 30: 2114-2120. doi:10.1093/bioinformatics/btu170

Brick K, Smagulova F, Khil P, Camerini-Otero RD, Petukhova GV. 2012. Genetic recombination is directed away from functional genomic elements in mice. Nature 485: 642-645. doi:10 $.1038 /$ nature 11089

Buard J, Barthès P, Grey C, de Massy B. 2009. Distinct histone modifications define initiation and repair of meiotic recombi- nation in the mouse. $E M B O /$ 28: 2616-2624. doi:10.1038/ emboj.2009.207

Buenrostro JD, Giresi PG, Zaba LC, Chang HY, Greenleaf WJ. 2013. Transposition of native chromatin for fast and sensitive epigenomic profiling of open chromatin, DNA-binding proteins and nucleosome position. Nat Methods 10: 1213-1218. doi:10.1038/nmeth. 2688

Choi K, Zhao X, Kelly KA, Venn O, Higgins JD, Yelina NE, Hardcastle TJ, Ziolkowski PA, Copenhaver GP, Franklin FC, et al. 2013. Arabidopsis meiotic crossover hot spots overlap with H2A.Z nucleosomes at gene promoters. Nat Genet 45: 1327-1336. doi:10.1038/ng.2766

Clapier CR, Cairns BR. 2009. The biology of chromatin remodeling complexes. Annu Rev Biochem 78: 273-304. doi:10.1146/ annurev.biochem.77.062706.153223

Clapier CR, Iwasa J, Cairns BR, Peterson CL. 2017. Mechanisms of action and regulation of ATP-dependent chromatin-remodelling complexes. Nat Rev Mol Cell Biol 18: 407-422. doi:10 $.1038 / \mathrm{nrm} .2017 .26$

Cobb J, Cargile B, Handel MA. 1999. Acquisition of competence to condense metaphase I chromosomes during spermatogenesis. Dev Biol 205: 49-64. doi:10.1006/dbio.1998.9101

Corces MR, Buenrostro JD, Wu B, Greenside PG, Chan SM, Koenig JL, Snyder MP, Pritchard JK, Kundaje A, Greenleaf WJ, et al. 2016. Lineage-specific and single-cell chromatin accessibility charts human hematopoiesis and leukemia evolution. Nat Genet 48: 1193-1203. doi:10.1038/ng.3646

De La Fuente R, Baumann C, Fan T, Schmidtmann A, Dobrinski I, Muegge K. 2006. Lsh is required for meiotic chromosome synapsis and retrotransposon silencing in female germ cells. Nat Cell Biol 8: 1448-1454. doi:10.1038/ncb1513

Diagouraga B, Clément JAJ, Duret L, Kadlec J, de Massy B, Baudat F. 2018. PRDM9 methyltransferase activity is essential for meiotic DNA double-strand break formation at its binding sites. Mol Cell 69: 853-865.e6. doi:10.1016/j.molcel.2018.01 .033

Eram MS, Bustos SP, Lima-Fernandes E, Siarheyeva A, Senisterra G, Hajian T, Chau I, Duan S, Wu H, Dombrovski L, et al. 2014. Trimethylation of histone H3 lysine 36 by human methyltransferase PRDM9 protein. I Biol Chem 289: 12177-12188. doi:10.1074/jbc.M113.523183

Ernst J, Kellis M. 2012. ChromHMM: automating chromatinstate discovery and characterization. Nat Methods 9: 215216. doi:10.1038/nmeth.1906

Geiman TM, Tessarollo L, Anver MR, Kopp JB, Ward JM, Muegge K. 2001. Lsh, a SNF2 family member, is required for normal murine development. Biochim Biophys Acta 1526: 211-220. doi:10.1016/S0304-4165(01)00129-5

Grey C, Clément JA, Buard J, Leblanc B, Gut I, Gut M, Duret L, de Massy B. 2017. In vivo binding of PRDM9 reveals interactions with noncanonical genomic sites. Genome Res 27: 580-590. doi:10.1101/gr.217240.116

Hayashi K, Yoshida K, Matsui Y. 2005. A histone H3 methyltransferase controls epigenetic events required for meiotic prophase. Nature 438: 374-378. doi:10.1038/nature04112

Heinz S, Romanoski CE, Benner C, Allison KA, Kaikkonen MU, Orozco LD, Glass CK. 2013. Effect of natural genetic variation on enhancer selection and function. Nature 503: 487-492. doi:10.1038/nature12615

Hinch AG, Zhang G, Becker PW, Moralli D, Hinch R, Davies B, Bowden R, Donnelly P. 2019. Factors influencing meiotic recombination revealed by whole-genome sequencing of single sperm. Science 363: eaau8861. doi:10.1126/science.aau8861

Houle AA, Gibling H, Lamaze FC, Edgington HA, Soave D, Fave MJ, Agbessi M, Bruat V, Stein LD, Awadalla P. 2018. Aberrant 
PRDM9 expression impacts the pan-cancer genomic landscape. Genome Res 28: 1611-1620. doi:10.1101/gr.231696 .117

Iwafuchi-Doi M, Zaret KS. 2014. Pioneer transcription factors in cell reprogramming. Genes Dev 28: 2679-2692. doi:10.1101/ gad.253443.114

Jenness C, Giunta S, Müller MM, Kimura H, Muir TW, Funabiki H. 2018. HELLS and CDCA7 comprise a bipartite nucleosome remodeling complex defective in ICF syndrome. Proc Nat1 Acad Sci 115: E876-E885. doi:10.1073/pnas.1717509115

Jung M, Wells D, Rusch J, Ahmad S, Marchini J, Myers SR, Conrad DF. 2019. Unified single-cell analysis of testis gene regulation and pathology in five mouse strains. Elife 8: e43966. doi:10.7554/eLife.43966

Keeney S. 2001. Mechanism and control of meiotic recombination initiation. Curr Top Dev Biol 52: 1-53. doi:10.1016/ S0070-2153(01)52008-6

Khil PP, Smagulova F, Brick KM, Camerini-Otero RD, Petukhova GV. 2012. Sensitive mapping of recombination hotspots using sequencing-based detection of ssDNA. Genome Res 22: 957965. doi:10.1101/gr.130583.111

King HW, Klose RJ. 2017. The pioneer factor OCT4 requires the chromatin remodeller BRG1 to support gene regulatory element function in mouse embryonic stem cells. Elife 6: e22631. doi:10.7554/eLife.22631

Kono H, Tamura M, Osada N, Suzuki H, Abe K, Moriwaki K, Ohta K, Shiroishi T. 2014. Prdm9 polymorphism unveils mouse evolutionary tracks. DNA Res 21: 315-326. doi:10 $.1093 /$ dnares/dst059

Lam KG, Brick K, Cheng G, Pratto F, Camerini-Otero RD. 2019. Cell-type-specific genomics reveals histone modification dynamics in mammalian meiosis. Nat Commun 10: 3821. doi:10.1038/s41467-019-11820-7

Lange J, Yamada S, Tischfield SE, Pan J, Kim S, Zhu X, Socci ND, Jasin M, Keeney S. 2016. The landscape of mouse meiotic double-strand break formation, processing, and repair. Cell 167: 695-708.e16. doi:10.1016/j.cell.2016.09.035

Langmead B, Trapnell C, Pop M, Salzberg SL. 2009. Ultrafast and memory-efficient alignment of short DNA sequences to the human genome. Genome Biol 10: R25. doi:10.1186/gb-200910-3-r25

Lex A, Gehlenborg N, Strobelt H, Vuillemot R, Pfister H. 2014. UpSet: visualization of intersecting sets. IEEE Trans Vis Comput Graph 20: 1983-1992. doi:10.1109/TVCG.2014.2346248

Li H, Durbin R. 2009. Fast and accurate short read alignment with Burrows-Wheeler transform. Bioinformatics 25: 1754-1760. doi:10.1093/bioinformatics/btp324

Li M, Huang T, Li MJ, Zhang CX, Yu XC, Yin YY, Liu C, Wang X, Feng HW, Zhang T, et al. 2019. The histone modification reader ZCWPW1 is required for meiosis prophase I in male but not in female mice. Sci $A d v$ 5: eaax1101. doi:10.1126/sciadv .aax1101

Maezawa S, Yukawa M, Alavattam KG, Barski A, Namekawa SH. 2018. Dynamic reorganization of open chromatin underlies diverse transcriptomes during spermatogenesis. Nucleic Acids Res 46: 593-608. doi:10.1093/nar/gkx1052

Meuleman W, Peric-Hupkes D, Kind J, Beaudry JB, Pagie L, Kellis M, Reinders M, Wessels L, van Steensel B. 2013. Constitutive nuclear lamina-genome interactions are highly conserved and associated with A/T-rich sequence. Genome Res 23: 270-280. doi:10.1101/gr.141028.112

Mihola O, Pratto F, Brick K, Linhartova E, Kobets T, Flachs P, Baker CL, Sedlacek R, Paigen K, Petkov PM, et al. 2019. Histone methyltransferase PRDM9 is not essential for meiosis in male mice. Genome Res 29: 1078-1086. doi:10.1101/gr.244426.118
Myers S, Bowden R, Tumian A, Bontrop RE, Freeman C, MacFie TS, McVean G, Donnelly P. 2010. Drive against hotspot motifs in primates implicates the PRDM9 gene in meiotic recombination. Science 327: 876-879. doi:10.1126/science.1182363

Neale MJ, Keeney S. 2006. Clarifying the mechanics of DNA strand exchange in meiotic recombination. Nature 442: 153158. doi: $10.1038 /$ nature 04885

Ou J, Liu H, Yu J, Kelliher MA, Castilla LH, Lawson ND, Zhu LJ. 2018. ATACseqQC: a Bioconductor package for post-alignment quality assessment of ATAC-seq data. BMC Genomics 19: 169. doi:10.1186/s12864-018-4559-3

Papamichos-Chronakis M, Watanabe S, Rando OJ, Peterson CL. 2011. Global regulation of H2A.Z localization by the INO80 chromatin-remodeling enzyme is essential for genome integrity. Cell 144: 200-213. doi:10.1016/j.cell.2010.12.021

Parvanov ED, Petkov PM, Paigen K. 2010. Prdm9 controls activation of mammalian recombination hotspots. Science 327: 835. doi:10.1126/science.1181495

Parvanov ED, Tian H, Billings T, Saxl RL, Spruce C, Aithal R, Krejci L, Paigen K, Petkov PM. 2017. PRDM9 interactions with other proteins provide a link between recombination hotspots and the chromosomal axis in meiosis. Mol Biol Cell 28: 488-499. doi:10.1091/mbc.e16-09-0686

Patel L, Kang R, Rosenberg SC, Qiu Y, Raviram R, Chee S, Hu R, Ren B, Cole F, Corbett KD. 2019. Dynamic reorganization of the genome shapes the recombination landscape in meiotic prophase. Nat Struct Mol Biol 26: 164-174. doi:10.1038/ s41594-019-0187-0

Peters AH, Plug AW, van Vugt MJ, de Boer P. 1997. A drying-down technique for the spreading of mammalian meiocytes from the male and female germline. Chromosome Res 5: 66-68. doi:10.1023/A:1018445520117

Price BD, D'Andrea AD. 2013. Chromatin remodeling at DNA double-strand breaks. Cell 152: 1344-1354. doi:10.1016/j.cell .2013.02.011

Quinlan AR, Hall IM. 2010. BEDTools: a flexible suite of utilities for comparing genomic features. Bioinformatics 26: 841-842. doi:10.1093/bioinformatics/btq033

Raghupathy N, Choi K, Vincent MJ, Beane GL, Sheppard KS, Munger SC, Korstanje R, Pardo-Manual de Villena F, Churchill GA. 2018. Hierarchical analysis of RNA-seq reads improves the accuracy of allele-specific expression. Bioinformatics 34: 2177-2184. doi:10.1093/bioinformatics/ bty078

Ren J, Briones V, Barbour S, Yu W, Han Y, Terashima M, Muegge K. 2015. The ATP binding site of the chromatin remodeling homolog Lsh is required for nucleosome density and de novo DNA methylation at repeat sequences. Nucleic Acids Res 43: 1444-1455. doi:10.1093/nar/gku1371

Robinson MD, McCarthy DJ, Smyth GK. 2010. edgeR: a Bioconductor package for differential expression analysis of digital gene expression data. Bioinformatics 26: 139-140. doi:10 .1093/bioinformatics/btp616

Sadate-Ngatchou PI, Payne CJ, Dearth AT, Braun RE. 2008. Cre recombinase activity specific to postnatal, premeiotic male germ cells in transgenic mice. Genesis 46: 738-742. doi:10 $.1002 / \operatorname{dvg} .20437$

Saldanha AJ. 2004. Java Treeview-extensible visualization of microarray data. Bioinformatics 20: 3246-3248. doi:10.1093/ bioinformatics/bth349

Serber DW, Runge JS, Menon DU, Magnuson T. 2016. The mouse INO80 chromatin-remodeling complex is an essential meiotic factor for spermatogenesis. Biol Reprod 94: 8. doi:10.1095/biol reprod. 115.135533 
Shen Y, Yue F, McCleary DF, Ye Z, Edsall L, Kuan S, Wagner U, Dixon J, Lee L, Lobanenkov VV, et al. 2012. A map of the cis-regulatory sequences in the mouse genome. Nature 488: 116-120. doi:10.1038/nature11243

Shenkar R, Shen MH, Arnheim N. 1991. DNase I-hypersensitive sites and transcription factor-binding motifs within the mouse E $\beta$ meiotic recombination hot spot. Mol Cell Biol 11: 1813-1819. doi:10.1128/MCB.11.4.1813

Smagulova F, Gregoretti IV, Brick K, Khil P, Camerini-Otero RD, Petukhova GV. 2011. Genome-wide analysis reveals novel molecular features of mouse recombination hotspots. Nature 472: 375-378. doi:10.1038/nature09869

Smagulova F, Brick K, Pu Y, Camerini-Otero RD, Petukhova GV. 2016. The evolutionary turnover of recombination hot spots contributes to speciation in mice. Genes Dev 30: 266-280. doi:10.1101/gad.270009.115

Soufi A, Donahue G, Zaret KS. 2012. Facilitators and impediments of the pluripotency reprogramming factors' initial engagement with the genome. Cell 151: 994-1004. doi:10 $.1016 /$ j.cell.2012.09.045

Striedner Y, Schwarz T, Welte T, Futschik A, Rant U, TiemannBoege I. 2017. The long zinc finger domain of PRDM9 forms a highly stable and long-lived complex with its DNA recognition sequence. Chromosome Res 25: 155-172. doi:10.1007/ s10577-017-9552-1

Sun F, Fujiwara Y, Reinholdt LG, Hu J, Saxl RL, Baker CL, Petkov PM, Paigen K, Handel MA. 2015. Nuclear localization of PRDM9 and its role in meiotic chromatin modifications and homologous synapsis. Chromosoma 124: 397-415. doi:10 .1007/s00412-015-0511-3

Sung MH, Guertin MJ, Baek S, Hager GL. 2014. DNase footprint signatures are dictated by factor dynamics and DNA sequence. Mol Cell 56: 275-285. doi:10.1016/j.molcel.2014.08 .016

Swinstead EE, Miranda TB, Paakinaho V, Baek S, Goldstein I, Hawkins M, Karpova TS, Ball D, Mazza D, Lavis LD, et al. 2016. Steroid receptors reprogram FoxAl occupancy through dynamic chromatin transitions. Cell 165: 593-605. doi:10 $.1016 /$ j.cell.2016.02.067

Termanis A, Torrea N, Culley J, Kerr A, Ramsahoye B, Stancheva I. 2016. The SNF2 family ATPase LSH promotes cell-autonomous de novo DNA methylation in somatic cells. Nucleic Acids Res 44: 7592-7604. doi:10.1093/nar/gkw424

Thijssen PE, Ito Y, Grillo G, Wang J, Velasco G, Nitta H, Unoki M, Yoshihara M, Suyama M, Sun Y, et al. 2015. Mutations in
CDCA7 and HELLS cause immunodeficiency-centromeric instability-facial anomalies syndrome. Nat Commun 6: 7870. doi:10.1038/ncomms 8870

Tock AJ, Henderson IR. 2018. Hotspots for initiation of meiotic recombination. Front Genet 9: 521. doi:10.3389/fgene.2018 .00521

von Eyss B, Maaskola J, Memczak S, Möllmann K, Schuetz A, Loddenkemper C, Tanh MD, Otto A, Muegge K, Heinemann $\mathrm{U}$, et al. 2012. The SNF2-like helicase HELLS mediates E2F3-dependent transcription and cellular transformation. EMBO / 31: 972-985. doi:10.1038/emboj.2011.451

Walker M, Billings T, Baker CL, Powers N, Tian H, Saxl RL, Choi K, Hibbs MA, Carter GW, Handel MA, et al. 2015. Affinity-seq detects genome-wide PRDM9 binding sites and reveals the impact of prior chromatin modifications on mammalian recombination hotspot usage. Epigenetics Chromatin 8: 31. doi:10.1186/s13072-015-0024-6

Wasserman WW, Sandelin A. 2004. Applied bioinformatics for the identification of regulatory elements. Nat Rev Genet 5: 276-287. doi:10.1038/nrg1315

Wu ZK, Getun IV, Bois PR. 2010. Anatomy of mouse recombination hot spots. Nucleic Acids Res 38: 2346-2354. doi:10.1093/ nar/gkp1251

Yamada S, Kugou K, Ding DQ, Fujita Y, Hiraoka Y, Murakami H, Ohta K, Yamada T. 2018. The histone variant H2A.Z promotes initiation of meiotic recombination in fission yeast. Nucleic Acids Res 46: 609-620. doi:10.1093/nar/gkx1110

Yu W, McIntosh C, Lister R, Zhu I, Han Y, Ren J, Landsman D, Lee E, Briones V, Terashima M, et al. 2014. Genome-wide DNA methylation patterns in LSH mutant reveals de-repression of repeat elements and redundant epigenetic silencing pathways. Genome Res 24: 1613-1623. doi:10.1101/gr .172015 .114

Zaret KS, Mango SE. 2016. Pioneer transcription factors, chromatin dynamics, and cell fate control. Curr Opin Genet Dev 37: 76-81. doi:10.1016/j.gde.2015.12.003

Zeng W, Baumann C, Schmidtmann A, Honaramooz A, Tang L, Bondareva A, Dores C, Fan T, Xi S, Geiman T, et al. 2011. Lymphoid-specific helicase (HELLS) is essential for meiotic progression in mouse spermatocytes. Biol Reprod 84: 12351241. doi:10.1095/biolreprod.110.085720

Zhang Y, Liu T, Meyer CA, Eeckhoute J, Johnson DS, Bernstein BE, Nusbaum C, Myers RM, Brown M, Li W, et al. 2008. Model-based analysis of ChIP-Seq (MACS). Genome Biol 9: R137. doi:10.1186/gb-2008-9-9-r137 


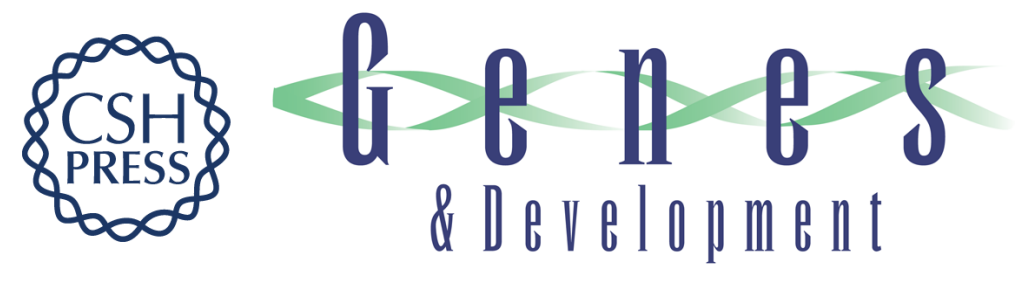

\section{HELLS and PRDM9 form a pioneer complex to open chromatin at meiotic recombination hot spots}

Catrina Spruce, Sibongakonke Dlamini, Guruprasad Ananda, et al.

Genes Dev. 2020, 34: originally published online January 30, 2020

Access the most recent version at doi:10.1101/gad.333542.119

\section{Supplemental http://genesdev.cshlp.org/content/suppl/2020/01/28/gad.333542.119.DC1 \\ Material}

Related Content Pioneering meiotic recombination

Kris G. Alavattam, Hironori Abe and Satoshi H. Namekawa

Genes Dev. March , 2020 34: 395-397

References This article cites 82 articles, 21 of which can be accessed free at:

http://genesdev.cshlp.org/content/34/5-6/398.full.html\#ref-list-1

Articles cited in:

http://genesdev.cshlp.org/content/34/5-6/398.full.html\#related-urls

Creative This article is distributed exclusively by Cold Spring Harbor Laboratory Press for the first Commons

License

six months after the full-issue publication date (see

http://genesdev.cshlp.org/site/misc/terms.xhtml). After six months, it is available under a Creative Commons License (Attribution-NonCommercial 4.0 International), as described at http://creativecommons.org/licenses/by-nc/4.0/.

Email Alerting

Service

Receive free email alerts when new articles cite this article - sign up in the box at the top right corner of the article or click here.

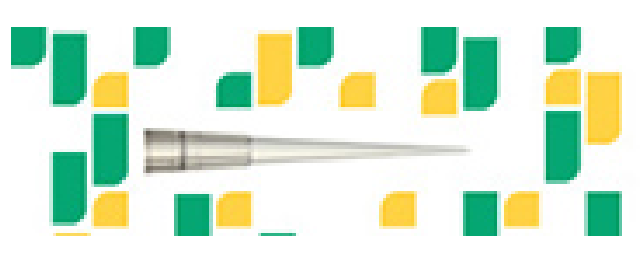

Focused on your science. 